\title{
From Verified Parameter Identification to the Design of Interval Observers and Cooperativity-Preserving Controllers - An Experimental Case Study
}

\author{
Andreas Rauh and Julia Kersten ${ }^{a}$
}

\begin{abstract}
One of the most important advantages of interval observers and the associated trajectory computation is their capability to provide estimates for a given dynamic system model in terms of guaranteed state bounds which are compatible with measured data subject to bounded uncertainty. However, the inevitable requirement for being able to produce such verified bounds is the knowledge about a dynamic system model in which possible uncertainties and inaccuracies are themselves represented by guaranteed bounds. For that reason, classical point-valued parameter identification schemes are often not sufficient or should, at least, be handled with sufficient care if safety critical applications are of interest. This paper provides an application-oriented description of the major steps leading from a control-oriented system model with an associated interval-valued parameter and disturbance identification to a verified design of interval observers which provide the basis for the development and implementation of cooperativity-preserving feedback controllers. Such combined control and observer structures allow for forecasting guaranteed lower and upper state bounds that can be determined by solving initial value problems for crisp-parameter models. As such, they replace the significantly more demanding task of computing tubes of reachable states by means of general-purpose interval methods. The corresponding computational steps for the cooperativity-preserving control and observer synthesis are described and visualized for the temperature control of a laboratory-scale test rig available at the Chair of Mechatronics at the University of Rostock.
\end{abstract}

Keywords: interval analysis, verified parameter identification, interval observers, cooperative system dynamics, interval-based feedback control

${ }^{a}$ University of Rostock, Chair of Mechatronics, Justus-von-Liebig-Weg 6, D-18059 Rostock, Germany E-mail: \{Andreas.Rauh, Julia.Kersten\}@uni-rostock.de 


\section{Introduction}

At least for the last two decades, interval observers have been designed for several different types of dynamic system models. Such system models can be characterized into continuous- and discrete-time state-space representations of systems with finite-dimensional dynamics as well as into special types of partial differential equations (PDEs) $[11,14,18,19,34,35,43]$. Especially for the case of finite-dimensional systems, linear time-invariant, linear parameter-varying, linear time-varying and (special types) of nonlinear dynamics have been accounted for $[6,24,40,41]$. If nonlinear dynamics are concerned, these system models are often described in terms of Takagi-Sugeno [26,52] or switched uncertain system models $[13,27,33,36]$, leading to a specific characterization of uncertainty in the entries of the system and input matrices by interpolations between various linear dynamic system models. For this type of models with bounded uncertainties in the system and input matrices, the aforementioned linear models serve as an embedding of the actual nonlinear behavior into an overapproximation by means of a convex polytope of extremal realizations which — by a suitable interpolation - account for either linear parameter-varying or linear time-varying models.

As shown in $[6,40,41]$, the design of interval observers can be simplified significantly if the dynamics under consideration follow specific monotonicity properties. In this frame, cooperativity of the state equations is of foremost interest. If this property is satisfied, it becomes possible to evaluate a finite number of independent bounding systems which lead to a description of worst-case lower and upper bounds for the sets of all reachable states. Sufficient requirements for cooperativity of a dynamic system were published, for example, in $[7,50]$. In contrast to sets of state equations in which lower and upper state bounds are fully coupled, the well-known wrapping effect $[15,28,30,31,37,39]$ arising in mostly any verified evaluation of an initial value problem (IVP) by means of interval analysis can be suppressed to a large extent if the system dynamics are ensured to be cooperative. For control applications, asymptotically stable dynamics (or respectively, input-to-state stability (ISS)) of the estimation errors of the bounding systems should be verified. Then, it can be guaranteed that the resulting bounds for the estimated state enclosures do not grow infinitely if the actual open- or closed-loop system behavior, from which the measured data are gathered, is evaluated by means of an interval observer.

Application scenarios in which interval observers have been applied successfully to dynamic systems in engineering as well as related fields such as computational biology are (without claim of completeness): vehicle lateral dynamics [12], state estimation of induction machines [25], control of the air-fuel ratio in direct injection combustion engines [5], state estimation in anaerobic digestion processes [1], exothermic fed-batch reactors [48], and other (bio-)technological processes with uncertain kinetics for growth of microorganisms and wastewater treatment $[9,10]$, fault detection in flight control systems [8], as well as fault detection and estimation algorithms on the basis of Takagi-Sugeno models [32]. Although the summary above was mainly focused on finite-dimensional system models, also sets of PDEs such as the parabolic differential equation of heat conduction were investigated. There, two 
fundamentally different approaches could be distinguished, namely, techniques relying on a replacement of the infinite-dimensional dynamics by a finite-dimensional system model before the observer design (early lumping) and the design of observers and its stability proof on the basis of the PDE model (late lumping) [18,20].

In most of these application-oriented references, the parameterization of the employed interval estimation schemes was based on the solution of systems of linear matrix inequalities (LMIs) [2,49] which allow for a constructive proof of the stability of the resulting error dynamics and help to turn the observer model into a cooperative form which, as mentioned above, allows for an independent evaluation of (usually two) mutually decoupled sets of bounding systems.

In contrast to the interval observers discussed above, also set-valued estimation techniques can be applied [21]. These techniques aim at predicting guaranteed state enclosures (via an interval evaluation of an IVP for the set of state equations) between two subsequent time instants at which measurements are available. As soon as new measured data exist, the predicted state enclosures are tightened by means of problem specific consistency tests allowing for an exclusion of subintervals of the predicted state enclosures (respectively, the predicted state boxes). These consistency tests rely on the technique on set inversion via interval analysis (SIVIA), as well as on contractors such as the Krawczyk iteration [15,23,38] or further interval Newton techniques. In contrast to the observer-based bounding approach, this type of estimation scheme is commonly much more computationally demanding because it usually relies on an interval subdivision during the state prediction and during the elimination of inconsistent state domains (cf. SIVIA). For that reason, these prediction-correction schemes are better suited for pure offline applications and for systems in a non-cooperative state-space representation, while real-time control scenarios as investigated in this paper usually make use of the observer-based framework, possibly after transforming the sets of state equations into a cooperative representation $[3,17]$.

Both, the observer-based estimation framework and the prediction-correction scheme, are model-based techniques. This means that both of them require an accurate mathematical system representation so that the estimation scheme built on top of the mathematical model can produce reliable estimation results. For that reason, this paper describes the complete development cycle of an interval observer-based feedback control synthesis, starting with the fundamental stage of verified parameter identification. Hence, the interaction of these three tasks is in the focus of this paper, while they were so far only investigated individually in the contributions $[42,44-46]$.

This paper is structured as follows. Sec. 2 presents verified, interval-based routines for the identification of a control-oriented system model for the distributed heating system serving as an application scenario in this paper. Based on the design of an interval observer, the dual task, namely, design of a cooperativitypreserving feedback controller is investigated in Sec. 3. Simulation results as well as an experimental validation complete this section, before conclusions and an outlook on future work are given in Sec. 4 . 


\section{Control-Oriented Finite-Volume Modeling of a Distributed Heating System with the Aim of an Interval Observer Design}

\subsection{Finite Volume Modeling}

Fig. 1 depicts the benchmark scenario with which the complete design cycle of an interval observer-based cooperativity-preserving feedback control synthesis is illustrated. The overall design starts with a suitable control-oriented modeling in terms of ordinary differential equations (ODEs) — which serve as an earlylumping approximation for the underlying infinite-dimensional PDE dynamics and a subsequent verified parameter identification. The experimental set-up $[42,45]$ consists of a metallic rod of length $l$, width $b$, and height $h,(b, h \ll l)$. In Fig. 1 , it is hidden behind the white Styrofoam insulation. For modeling, the thermal conductivity of the rod in one relevant space coordinate is denoted by $\lambda_{\mathrm{R}}$, its volume density by $\rho_{\mathrm{R}}$, and the specific heat capacity by $c_{\mathrm{R}}$. Furthermore, it is assumed that the temperature distribution in the rod is described in terms of the temperature difference $\vartheta(x, t):=\theta(x, t)-\theta_{\mathrm{A}}$, in which $\theta_{\mathrm{A}}$ denotes the temperature of the ambient air assumed to be constant in good approximation.

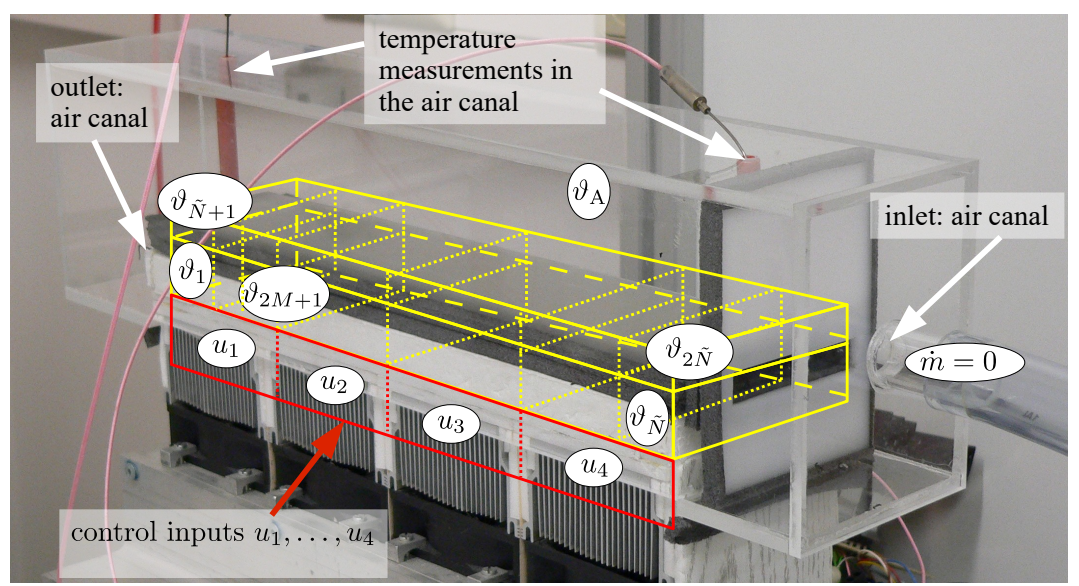

Figure 1: Test rig for the experimental, real-time capable validation of the intervalbased control and observer design.

According to $[42,45]$, where the state equations for the rod and air canal temperature were derived after discretizing the $\operatorname{rod}$ and air canal into each $\tilde{N}=$ $N \cdot(2 M+1), M \in \mathbb{N}_{0}$, finite volume elements with piece-wise homogeneous temperatures, the parameter-dependent linear state-space representation

$$
\dot{\mathbf{x}}(t)=\mathbf{A}(\mathbf{p}) \cdot \mathbf{x}(t)+\mathbf{B} \cdot \mathbf{u}(t)
$$


is obtained, where the state vector is denoted by $\mathbf{x}(t)=\left[\begin{array}{llll}\vartheta_{1}(t) & \ldots & \vartheta_{2 \tilde{N}}(t)\end{array}\right]^{T}$ and the control vector by $\mathbf{u}(t)=\left[\begin{array}{lll}u_{1}(t) & \ldots & u_{N}(t)\end{array}\right]^{T}$ (heat flows provided by $N=4$ Peltier elements). For this system model, a list of $L$ parameter intervals ${ }^{1}$ was identified by means of a verified simulation-based parameter identification $[42,45]$. A detailed analysis of the structure of the system matrix $\mathbf{A}(\mathbf{p})$ reveals the property that it is Hurwitz (describing asymptotically stable ${ }^{2}$ dynamics) and Metzler (containing only non-negative entries outside its diagonal) for any physically reasonable parameterization. These reasonable parameterizations correspond to $\alpha>0$ (heat transfer between rod and air canal), $\alpha_{\mathrm{B}}>0$ (heat transfer on the bottom of the rod), $\alpha_{\mathrm{T}}>0$ (heat transfer between air canal and ambiance), $\lambda_{\mathrm{R}}>0$ (heat conductance), and the correction terms $\Delta \alpha>0$ (accounting for the improved heat convection at the air canal outlet) as well as $\Delta m_{\mathrm{a}}>0$ (representing the enlarged thermal capacity at the air canal inlet segment).

Due to the fact that cooperativity [50] and positivity [16] of the set of ODEs (1) is ensured for element-wise non-negative products $\mathbf{B} \cdot \mathbf{u}(t)$, lower and upper bounds for all reachable state trajectories $\mathbf{x}(t)$ can be determined in this case by the bounding systems

$$
\mathbf{A}(\underline{\mathbf{p}}) \cdot \mathbf{v}(t)+\mathbf{B} \cdot \mathbf{u}(t)=\dot{\mathbf{v}}(t) \leq \dot{\mathbf{x}}(t) \leq \dot{\mathbf{w}}(t)=\mathbf{A}(\overline{\mathbf{p}}) \cdot \mathbf{w}(t)+\mathbf{B} \cdot \mathbf{u}(t)
$$

These bounding systems lead to guaranteed interval enclosures not only for the states themselves but also for the measurable system outputs $\mathbf{y}(t)$ according to

$$
\mathbf{x}(t) \in[\mathbf{x}](t)=[\mathbf{v}(t) ; \mathbf{w}(t)] \quad \text { and } \quad \mathbf{y}(t) \in[\mathbf{y}](t)=[\mathbf{C} \cdot \mathbf{v}(t) ; \mathbf{C} \cdot \mathbf{w}(t)]
$$

with element-wise non-negative states and non-negative entries in the output matrix $\mathbf{C}$. In detail, the output matrix $\mathbf{C}$ in the linear relation $\mathbf{y}(t)=\mathbf{C} \cdot \mathbf{x}(t)$ is zero except for exactly one element per row which is equal to one. It represents the sensor model of temperature measurements in the segment midpoints above each of the Peltier elements as well as at the air canal in- and outlet positions.

To describe the bounding systems in (2), all physical parameters are assumed to be contained in pairwise independent scalar intervals $\alpha \in[\underline{\alpha} ; \bar{\alpha}], \alpha_{\mathrm{B}} \in\left[\underline{\alpha}_{\mathrm{B}} ; \bar{\alpha}_{\mathrm{B}}\right]$, $\alpha_{\mathrm{T}} \in\left[\underline{\alpha}_{\mathrm{T}} ; \bar{\alpha}_{\mathrm{T}}\right], \Delta \alpha \in[\underline{\Delta \alpha} ; \overline{\Delta \alpha}], \Delta m_{\mathrm{a}} \in\left[\underline{\Delta m}_{\mathrm{a}} ; \overline{\Delta m}_{\mathrm{a}}\right]$, and $\lambda_{\mathrm{R}} \in\left[\underline{\lambda}_{\mathrm{R}} ; \bar{\lambda}_{\mathrm{R}}\right]$

\footnotetext{
${ }^{1}$ Here, $L$ is the final number of intervals after the experimental identification, where each of them is given as a box containing the six parameters to be identified experimentally. These boxes were determined by a sensitivity-based splitting of an initial search domain until $L$ mutually disjoint boxes were obtained. For a limitation of the computational complexity in control and observer implementation, small boxes were merged at the end of the identification so that stability properties of the bounding systems (2) are preserved. For details concerning the splitting and merging procedure, the reader is referred to $[42,45]$.

${ }^{2}$ The Gershgorin circle theorem, cf. [53], can be employed for a straightforward stability analysis of this system model with point-valued and sufficiently tight uncertain parameters. For further discussions about the use of this stability criterion as a constraint during an interval-based parameter identification, the reader is referred to [45].
} 
from which the parameterizations

$$
\underline{\mathbf{p}}=\left[\begin{array}{c}
-\left(\bar{\lambda}_{\mathrm{R}} \cdot \frac{A_{\mathrm{c}}}{l_{\mathrm{s}}}+\left(\bar{\alpha}_{\mathrm{B}}+\bar{\alpha}\right) \cdot A_{\mathrm{s}}\right) \\
\underline{\lambda}_{\mathrm{R}} \cdot \frac{A_{\mathrm{c}}}{l_{\mathrm{s}}} \\
-\left(2 \bar{\lambda}_{\mathrm{R}} \cdot \frac{A_{\mathrm{c}}}{l_{\mathrm{s}}}+\left(\bar{\alpha}_{\mathrm{B}}+\bar{\alpha}\right) \cdot A_{\mathrm{s}}\right) \\
\underline{\underline{\alpha}} \cdot A_{\mathrm{s}} \\
-\left(\bar{\alpha}_{\mathrm{T}}+\bar{\alpha}+\overline{\Delta \alpha} \cdot \delta_{\tilde{N}+i, \tilde{N}+1}\right) \cdot A_{\mathrm{s}} \\
\left(\frac{m_{\mathrm{a}}}{\tilde{N}} \cdot\left(1+\delta_{\tilde{N}+i, 2 \tilde{N}} \cdot \overline{\Delta m}_{\mathrm{a}}\right)\right)^{-1}
\end{array}\right] \text { and } \overline{\mathbf{p}}=\left[\begin{array}{c}
-\left(\underline{\lambda}_{\mathrm{R}} \cdot \frac{A_{\mathrm{c}}}{l_{\mathrm{s}}}+\left(\underline{\alpha}_{\mathrm{B}}+\underline{\alpha}\right) \cdot A_{\mathrm{s}}\right) \\
\bar{\lambda}_{\mathrm{R}} \cdot \frac{A_{\mathrm{c}}}{l_{\mathrm{s}}} \\
-\left(2 \underline{\lambda}_{\mathrm{R}} \cdot \frac{A_{\mathrm{c}}}{l_{\mathrm{s}}}+\left(\underline{\alpha}_{\mathrm{B}}+\underline{\alpha}\right) \cdot A_{\mathrm{s}}\right) \\
\bar{\alpha} \cdot A_{\mathrm{s}} \\
-\left(\underline{\alpha}_{\mathrm{T}}+\underline{\alpha}+\underline{\Delta} \cdot \delta_{\tilde{N}+i, \tilde{N}+1}\right) \cdot A_{\mathrm{s}} \\
\left(\frac{m_{\mathrm{a}}}{\tilde{N}} \cdot\left(1+\delta_{\tilde{N}+i, 2 \tilde{N}} \cdot \underline{\Delta m_{\mathrm{a}}}\right)\right)^{-1}
\end{array}\right]
$$

are extracted so that the relation

$$
\mathbf{A}(\underline{\mathbf{p}}) \leq \mathbf{A}(\mathbf{p}) \leq \mathbf{A}(\overline{\mathbf{p}})
$$

holds in an element-wise manner. Further, geometrically a-priori known parameters that are included in (4) are the segment length $l_{\mathrm{s}}=\frac{l}{\tilde{N}}$, the vertical cross section area $A_{\mathrm{s}}=b \cdot h$ of the rod, and the effective surface $A_{\mathrm{s}}=b \cdot l_{\mathrm{s}}$ over which heat convection takes place; $m_{\mathrm{a}}$ is the overall air mass in the canal under the assumption of a constant ambient pressure with incompressible air.

\subsection{Interval-Based Parameter Identification}

For the purpose of the interval-based identification of the cooperative system model summarized in the previous subsection, the following assumptions have to be made:

- measured data $\mathbf{y}_{\mathrm{m}}$ are available at discrete points of time $t_{k}$,

- worst-case bounds $\left[-\Delta \mathbf{y}_{\mathrm{m}} ; \Delta \mathbf{y}_{\mathrm{m}}\right]$ for the measurement tolerances are known a-priori (as a model for sensor inaccuracies) according to

$$
\left[\mathbf{y}_{\mathrm{m}}\right]\left(t_{k}\right)=\mathbf{y}_{\mathrm{m}}\left(t_{k}\right)+\left[-\Delta \mathbf{y}_{\mathrm{m}} ; \Delta \mathbf{y}_{\mathrm{m}}\right],
$$

- the model to be parameterized is assumed to be structurally correct,

- outer enclosures for the domains of possibly uncertain initial states, i.e., v(0) and $\mathbf{w}(0)$, are given, and

- interval bounds $[\mathbf{p}]$ on the uncertain parameters are known in terms of conservative overapproximations.

To exclude parts of the domains of uncertain initial states and parts of the apriori given parameter intervals, a subdivision procedure of the respective domains is executed [4]. Based on the intersection of directly measured output intervals $\left[\mathbf{y}_{\mathrm{m}}\right]\left(t_{k}\right)$ and the respective interval-based simulation results $[\mathbf{y}]\left(t_{k}\right)$ for the IVPs consisting of Eqs. (2)-(5), the following cases are distinguished [42,47] in a SIVIAlike identification routine [15]:

Case 1: Parameter subintervals are yet undecided if their corresponding predicted output intervals overlap at least partially for all of the available sensors $i \in$ $\{1, \ldots, \operatorname{dim}\{\mathbf{y}\}\}$ at each point of time $t_{k}$ at which measurements are available and are never outside the range of measured data, i.e., $\left[y_{i}\right]\left(t_{k}\right) \nsubseteq\left[y_{\mathrm{m}, i}\right]\left(t_{k}\right)$ 
for at least one $i$ and $k$ with $\left[y_{\mathrm{m}, i}\right]\left(t_{k}\right) \cap\left[y_{i}\right]\left(t_{k}\right) \neq \emptyset$ for all $i$ and $k$. The corresponding parameter intervals need to be subdivided further in order to make a decision about feasibility.

Case 2: A parameter subinterval is guaranteed to be consistent with the measurements and the system structure if the corresponding output intervals are true subintervals of the interval-valued measured data for each of the available sensors $i$ at each point of time $t_{k}$, i.e., $\left[y_{i}\right]\left(t_{k}\right) \subseteq\left[y_{\mathrm{m}, i}\right]\left(t_{k}\right)$ for all $i$ and $k$. Such parameter subintervals are no longer investigated but stored in a list of guaranteed admissible boxes.

Case 3: A parameter subinterval is guaranteed to be inconsistent if there exists at least one point of time $t_{k}$ at which the computed output interval lies fully outside the range of the interval-valued measured data for at least one of the available sensors $i$, i.e., $\left[y_{\mathrm{m}, i}\right]\left(t_{k}\right) \cap\left[y_{i}\right]\left(t_{k}\right)=\emptyset$. The corresponding parameter subinterval is excluded from further evaluations and the underlying simulation is aborted as soon as the inconsistency is detected.

At the end of the subdivision-based parameter identification, a list of $L$ interval boxes exists. This number can be reduced by merging intervals if their union leads to a small increase of the parameters' volume [22] under the restriction that the asymptotic stability property of the bounding systems (2) must not be lost. The result of this merging is described in Sec. 2.5 of this paper.

\subsection{Fundamental Observer Approach}

For the cooperative model (2), representing a verified open-loop bounding system for all reachable states, an interval observer can be defined according to ${ }^{3}$

$$
\mathbf{A}_{\mathrm{O}}(\underline{\mathbf{p}}) \cdot \hat{\mathbf{v}}+\mathbf{B} \cdot \mathbf{u}+\mathbf{H} \cdot \underline{\mathbf{y}}_{\mathrm{m}}=\dot{\hat{\mathbf{v}}} \leq \dot{\hat{\mathbf{x}}} \leq \dot{\hat{\mathbf{w}}}=\mathbf{A}_{\mathrm{O}}(\overline{\mathbf{p}}) \cdot \hat{\mathbf{w}}+\mathbf{B} \cdot \mathbf{u}+\mathbf{H} \cdot \overline{\mathbf{y}}_{\mathrm{m}} .
$$

In (7), the system matrix

$$
\mathbf{A}_{\mathrm{O}}(\mathbf{p})=\mathbf{A}(\mathbf{p})-\mathbf{H C}=\mathbf{A}(\mathbf{p})-\mathcal{H}
$$

needs to be parameterized by choosing the gain $\mathbf{H}$ so that $\mathbf{A}_{\mathrm{O}}(\mathbf{p})$ is Hurwitz for all possible parameters $\mathbf{p}$. This property ensures asymptotic stability (respectively, ISS) of the error dynamics which can be verified if a parameter-independent, positive definite solution $\mathbf{P}_{\mathrm{O}}=\mathbf{P}_{\mathrm{O}}^{T} \succ 0$ exists for the matrix inequalities

$$
\mathbf{A}_{\mathrm{O}}(\underline{\mathbf{p}}) \cdot \mathbf{P}_{\mathrm{O}}+\mathbf{P}_{\mathrm{O}} \cdot \mathbf{A}_{\mathrm{O}}^{T}(\underline{\mathbf{p}}) \prec 0 \quad \text { and } \quad \mathbf{A}_{\mathrm{O}}(\overline{\mathbf{p}}) \cdot \mathbf{P}_{\mathrm{O}}+\mathbf{P}_{\mathrm{O}} \cdot \mathbf{A}_{\mathrm{O}}^{T}(\overline{\mathbf{p}}) \prec 0 .
$$

In addition, the matrix $\mathbf{H}$ needs to be constrained so that $\mathbf{A}_{\mathrm{O}}(\mathbf{p})$ is Metzler with

$$
A_{\mathrm{O}, i, j}(\mathbf{p}) \geq 0 \quad \text { for all } \quad i, j \in\{1, \ldots, 2 \tilde{N}\}, \quad i \neq j
$$

\footnotetext{
${ }^{3}$ For the sake of compactness, time arguments are omitted subsequently.
} 
as it was the case for the open-loop dynamics $\mathbf{A}(\mathbf{p})$. If (10) is fulfilled, the lower and upper bounds $\hat{\mathbf{v}}$ and $\hat{\mathbf{w}}$ of the observer estimates $(7)$ can be computed independently and represent guaranteed state bounds under consideration of the uncertain measurements ${ }^{4}$. As described in [42], a trivial choice for an observer gain $\mathbf{H}$ with sparse structure fulfilling both requirements (9) and (10), is given by

$$
\mathbf{H}=(\mathcal{K} \mathbf{C})^{T} \quad \text { with } \quad \mathcal{K}=\operatorname{diag}\{\boldsymbol{\kappa}\} \quad \text { and } \quad \boldsymbol{\kappa}=\left[\begin{array}{lll}
\kappa_{1} & \ldots & \kappa_{N+2}
\end{array}\right],
$$

$\kappa_{i}>0, i \in\{1, \ldots, N+2\}$. Defining the matrix $\mathcal{H}=\mathbf{H C}=\mathbf{C}^{T} \mathcal{K} \mathbf{C}$ with

$$
\mathcal{H}_{i, j}= \begin{cases}\kappa_{i} & \text { for } i=j=(\xi \cdot(2 M+1)-M), \xi \in\{1, \ldots, N\} \\ \kappa_{i} & \text { for } i=j, j \in\{\tilde{N}+1,2 \tilde{N}\} \\ 0 \quad \text { else }\end{cases}
$$

it can be shown that this choice leads to a pure adaptation of the diagonal entries of the system matrices in the state observer. Possibilities for a further optimization of the parameters $\kappa_{i}$ in this restricted observer structure as well as an optimization of all entries in a non-sparse solution for $\mathbf{H}$ with generally $2 \tilde{N} \cdot(N+1)=2 N$. $(N+2) \cdot(2 M+1)$ non-zero entries are given in the following subsection.

\subsection{Optimized Observer Parameterization}

To find optimal observer gains, which do not only ensure stability and cooperativity of the error dynamics, the following approach reduces the sensitivity of the estimation results with respect to unmodeled errors by using an $H_{\infty}$ synthesis. For that purpose, the error vector

$$
\mathbf{e}=\left[\begin{array}{ll}
(\hat{\mathbf{v}}-\mathbf{v})^{T} & (\hat{\mathbf{w}}-\mathbf{w})^{T}
\end{array}\right]^{T}
$$

is introduced to quantify the difference between the estimated and true lower and upper state bounds, respectively. Using the vector (13), the ODEs

$$
\dot{\mathbf{e}}=\left[\begin{array}{cc}
\mathbf{A}(\underline{\mathbf{p}})-\mathbf{H C} & \mathbf{0} \\
\mathbf{0} & \mathbf{A}(\overline{\mathbf{p}})-\mathbf{H C}
\end{array}\right] \mathbf{e}+\left[\begin{array}{l}
\mathbf{H} \\
\mathbf{H}
\end{array}\right] \boldsymbol{\zeta}
$$

for the estimation errors can be defined with the measurement tolerance vector $\boldsymbol{\zeta}$, $\zeta \in\left[-\Delta \mathbf{y}_{\mathrm{m}} ; \Delta \mathbf{y}_{\mathrm{m}}\right]$ in (6). Now, the augmented system output

$$
\mathbf{y}_{\infty}=\left[\begin{array}{cc}
\mathbf{0}_{(N+2) \times \tilde{N}} & \mathbf{0}_{(N+2) \times \tilde{N}} \\
-\nu \cdot \mathbf{I}_{\tilde{N} \times \tilde{N}} & \nu \cdot \mathbf{I}_{\tilde{N} \times \tilde{N}}
\end{array}\right] \mathbf{e}+\left[\begin{array}{c}
\mathbf{I}_{(N+2) \times(N+2)} \\
\mathbf{0}_{\tilde{N} \times(N+2)}
\end{array}\right] \boldsymbol{\zeta}=\mathbf{C}_{\infty} \mathbf{e}+\mathbf{D}_{\infty 1} \boldsymbol{\zeta}
$$

can be employed for a comparison of the influence of measurement errors $\boldsymbol{\zeta}$ with the weighted diameter $\nu \cdot(\hat{\mathbf{w}}-\mathbf{w})-\nu \cdot(\hat{\mathbf{v}}-\mathbf{v}), \nu>0$, between the open-loop state

\footnotetext{
${ }^{4}$ In this paper, we restrict ourselves to a quasi-continuous observer design despite the discretetime nature of measured data. This is admissible if the sampling intervals are shorter by at least one order of magnitude than the smallest time constant of the open- and closed-loop dynamics.
} 
bounds and the results of the interval observer. To solve this optimization task, the matrix inequality (cf. [45])

$$
\mathcal{L}(\boldsymbol{\Theta}):=\left[\begin{array}{ccc}
\boldsymbol{\Theta} & \mathbf{H} & \mathbf{P}_{\mathrm{O}} \mathbf{C}_{\infty}^{T} \\
\mathbf{H}^{T} & -\mathbf{I} & \mathbf{D}_{\infty 1}^{T} \\
\mathbf{C}_{\infty} \mathbf{P}_{\mathrm{O}} & \mathbf{D}_{\infty 1} & -\gamma_{\infty}^{2} \mathbf{I}
\end{array}\right] \prec 0 \quad \text { with } \quad \boldsymbol{\Theta} \in\{\underline{\boldsymbol{\Theta}}, \overline{\boldsymbol{\Theta}}\}
$$

is defined first for the two extremal systems

$$
\underline{\boldsymbol{\Theta}}:=\mathbf{A}_{\mathrm{O}}(\underline{\mathbf{p}}) \cdot \mathbf{P}_{\mathrm{O}}+\mathbf{P}_{\mathrm{O}} \cdot \mathbf{A}_{\mathrm{O}}^{T}(\underline{\mathbf{p}}) \quad \text { and } \quad \overline{\boldsymbol{\Theta}}:=\mathbf{A}_{\mathrm{O}}(\overline{\mathbf{p}}) \cdot \mathbf{P}_{\mathrm{O}}+\mathbf{P}_{\mathrm{O}} \cdot \mathbf{A}_{\mathrm{O}}^{T}(\overline{\mathbf{p}}) .
$$

Here, $\mathbf{P}_{\mathrm{O}}=\mathbf{P}_{\mathrm{O}}^{T} \succ 0$ specifies a quadratic Lyapunov function candidate, simultaneously valid for both $\underline{\boldsymbol{\Theta}}$ and $\overline{\boldsymbol{\Theta}}$. Then, a linearizing change of variables

$$
\mathbf{Q}_{\mathrm{O}}=\mathbf{Q}_{\mathrm{O}}^{T}=\mathbf{P}_{\mathrm{O}}^{-1} \succ 0 \text { with } \mathbf{Y}_{\mathrm{O}}^{T}=\mathbf{Q}_{\mathrm{O}} \mathbf{H}=\mathbf{P}_{\mathrm{O}}^{-1} \mathbf{H}
$$

leads to the design LMIs

$$
\mathcal{M}(\boldsymbol{\Sigma}):=\left[\begin{array}{ccc}
\boldsymbol{\Sigma} & \mathbf{Y}_{\mathrm{O}}^{T} & \mathbf{C}_{\infty}^{T} \\
\mathbf{Y}_{\mathrm{O}} & -\mathbf{I} & \mathbf{D}_{\infty 1}^{T} \\
\mathbf{C}_{\infty} & \mathbf{D}_{\infty 1} & -\mu_{\infty} \mathbf{I}
\end{array}\right] \prec 0 \quad \text { with } \quad \boldsymbol{\Sigma} \in\{\underline{\boldsymbol{\Sigma}}, \overline{\boldsymbol{\Sigma}}\}
$$

where

$$
\begin{aligned}
& \underline{\boldsymbol{\Sigma}}:=\mathbf{Q}_{\mathrm{O}} \mathbf{A}(\underline{\mathbf{p}})-\mathbf{Y}_{\mathrm{O}}^{T} \mathbf{C}+\mathbf{A}^{T}(\underline{\mathbf{p}}) \mathbf{Q}_{\mathrm{O}}-\mathbf{C}^{T} \mathbf{Y}_{\mathrm{O}}, \\
& \overline{\boldsymbol{\Sigma}}:=\mathbf{Q}_{\mathrm{O}} \mathbf{A}(\overline{\mathbf{p}})-\mathbf{Y}_{\mathrm{O}}^{T} \mathbf{C}+\mathbf{A}^{T}(\overline{\mathbf{p}}) \mathbf{Q}_{\mathrm{O}}-\mathbf{C}^{T} \mathbf{Y}_{\mathrm{O}},
\end{aligned}
$$

and

$$
\mu_{\infty}:=\gamma_{\infty}^{2} \geq 0
$$

hold. During the observer parameterization, which ensures ISS due to the inclusion of (20) in the design LMIs, the parameter $\mu_{\infty}$ is minimized. Cooperativity of the error dynamics is obtained by the element-wise defined inequality constraint

$$
\operatorname{col}\left(\left(\mathbf{A}(\mathbf{p})-\check{\mathbf{Q}}_{\mathrm{O}}^{-1} \mathbf{Y}_{\mathrm{O}}^{T} \mathbf{C}\right) \circ(\mathbf{E}-\mathbf{I})\right) \geq \mathbf{0}, \quad \mathbf{p} \in\{\underline{\mathbf{p}}, \overline{\mathbf{p}}\}
$$

where $\circ$ denotes the element-wise defined Hadamard product of two matrices with identical dimensions. In $(22)$, $\check{\mathbf{Q}}_{\mathrm{O}}$ is the result of the last iteration step of the solution of the LMI-based optimization problem. The iteration is stopped as soon as all constraints are satisfied and the norm between $\check{\mathbf{Q}}_{\mathrm{O}}$ and $\mathbf{Q}_{\mathrm{O}}$ falls below a pre-defined threshold value ${ }^{5}$.

This LMI-based optimization approach can be interpreted as a generalization of the straightforward parameterization mentioned in the previous subsection and provides a structurally optimal observer gain matrix $\mathbf{H}$ in a systematic manner.

\footnotetext{
${ }^{5}$ To prevent a poor convergence of the iterative minimization, the actual cost function is set to $\mu_{\infty}+\eta \cdot\left\|\mathbf{Q}_{\mathrm{O}}-\mathbf{Q}_{\mathrm{O}}\right\|$ with a sufficiently small penalty term $\eta>0$ as a generalization of (21).
} 
If simplified sparse solutions for $\mathbf{H}$ according to (11) shall be optimized by a minimization of $\mu_{\infty} \geq 0$ according to the constraint (21) with the help of the $H_{\infty}$ design described above, the LMIs $\mathcal{M}(\boldsymbol{\Sigma}) \prec 0$ in (19) with (20) turn into

$$
\mathcal{N}(\boldsymbol{\Xi}):=\left[\begin{array}{ccc}
\boldsymbol{\Xi} & \check{\mathbf{Q}} \cdot(\mathcal{K} \mathbf{C})^{T} & \mathbf{C}_{\infty}^{T} \\
(\mathcal{K} \mathbf{C}) \cdot \check{\mathbf{Q}} & -\mathbf{I} & \mathbf{D}_{\infty 1}^{T} \\
\mathbf{C}_{\infty} & \mathbf{D}_{\infty 1} & -\mu_{\infty} \mathbf{I}
\end{array}\right] \prec 0 \quad \text { for } \quad \boldsymbol{\Xi} \in\{\boldsymbol{\Xi}, \bar{\Xi}\}
$$

where

$$
\begin{aligned}
& \underline{\Xi}:=\mathbf{Q}_{\odot} \mathbf{A}(\underline{\mathbf{p}})-\check{\mathbf{Q}}_{\odot} \mathbf{C}^{T} \mathcal{K} \mathbf{C}+\mathbf{A}^{T}(\underline{\mathbf{p}}) \mathbf{Q}_{\mathrm{O}}-\mathbf{C}^{T} \mathcal{K} \mathbf{C} \check{\mathbf{Q}}_{\mathrm{O}}, \\
& \overline{\boldsymbol{\Xi}}:=\mathbf{Q}_{\mathrm{O}} \mathbf{A}(\overline{\mathbf{p}})-\check{\mathbf{Q}}_{\mathrm{O}} \mathbf{C}^{T} \mathcal{K} \mathbf{C}+\mathbf{A}^{T}(\overline{\mathbf{p}}) \mathbf{Q}_{\mathrm{O}}-\mathbf{C}^{T} \mathcal{K} \mathbf{C} \check{\mathbf{Q}}_{\mathrm{O}} .
\end{aligned}
$$

Hence, the following three options exist for the optimized cooperativity-preserving observer design:

O1 Determine the observer gain $\mathbf{H}$ as a solution of the LMIs (19) with (20) which minimizes the $H_{\infty}$ performance criterion (21) for the augmented output (15). To satisfy the Metzler property of the error dynamics, the constraint (22) is taken into account in an iterative manner, where $\mathbf{Q}_{\mathrm{O}}$ denotes the matrix $\mathbf{Q}_{\mathrm{O}}$ computed in the previous solution stage. In the first step, $\check{\mathbf{Q}}_{\mathrm{O}}$ is initialized by an identity matrix with appropriate dimensions.

O2 Determine optimal gains $\kappa_{i}>0$ in (11) as a solution of the LMIs (23) with (24) which minimize the $H_{\infty}$ performance criterion (21) for the output (15). In this case, the inequality constraint (22) is already satisfied due to the predefined structure of $\mathbf{H}$. Due to multiplicative couplings of $\mathbf{Q}_{\mathrm{O}}$ and $\mathcal{K}$ in (24), the solution is again determined iteratively.

O3 Determine optimal gain values $\kappa_{1}=\ldots=\kappa_{N+2}>0$ in analogy to the solution approach in option O2. Due to the fact that option O3 represents a constrained variant of $\mathbf{O 2}$ in the sense that all gains need to be identical, its solution can be used to initialize the iteration involved in option $\mathbf{O 2}$.

Practical experiments have shown that the convergence of the options $\mathbf{O} 2$ and O3 can be improved if the matrix $\check{\mathbf{Q}}_{\mathrm{O}}$ is updated by solving the feasibility problem related to (9) as soon as a new observer gain has been determined [45]. Note that each of these three options involves the solution of up to a few hundred iterations, where in each iteration LMI-based optimization routines are executed in a pure offline manner. For the application at hand, see also the following subsection, the respective state vector has the dimension $\mathbf{x} \in \mathbb{R}^{24}$.

\subsection{Implementation of a Parallel Bank of State Observers}

It is generally possible to perform the interval observer design and its implementation by two bounding systems (one for the lower and one for the upper state bounds) if stabilizing solutions for the design task exist. However, strong couplings 
between the matrix entries of $\mathbf{A}(\mathbf{p})$ and $\mathbf{A}(\overline{\mathbf{p}})$ can be caused by the underlying physical parameters such as the heat convection and heat conduction coefficients. These couplings can be captured in a much better way by the union over $L$ lower and upper bounding systems (corresponding to the outcome of the interval-based parameter identification) during the implementation of a parallel bank of observers. Here, the parameter list consists of $L$ mutually disjoint boxes except for selected edges and vertices. If these parameter boxes are bounded by the intervals

$$
\mathbf{p}_{\varsigma} \in\left[\underline{\mathbf{p}}_{\varsigma} ; \overline{\mathbf{p}}_{\varsigma}\right], \varsigma \in\{1, \ldots, L\}
$$

the associated parameter-dependent sets of ODEs are given by

$$
\dot{\mathbf{x}}_{\varsigma}=\mathbf{A}_{\varsigma} \mathbf{x}_{\varsigma}+\mathbf{B}_{\varsigma} \mathbf{u} \quad \text { with } \quad \mathbf{A}_{\varsigma} \in\left[\underline{\mathbf{A}}_{\varsigma} ; \overline{\mathbf{A}}_{\varsigma}\right] \quad \text { and } \quad \mathbf{B}_{\varsigma} \in\left[\underline{\mathbf{B}}_{\varsigma} ; \overline{\mathbf{B}}_{\varsigma}\right] .
$$

Using the cooperative open-loop models in Eq. (26), a bank of $L$ parallel interval observers can then be implemented on the basis of the uncertain measurements (6).

These observers provide the interval estimates

$$
\hat{\mathbf{x}} \in \bigcup_{\varsigma=1}^{L}\left[\hat{\mathbf{v}}_{\varsigma} ; \hat{\mathbf{w}}_{\varsigma}\right],
$$

which are usually tighter and, therefore, less conservative than the results from the previous subsection. The parameterization follows directly from the same approach as before, when a common gain $\mathbf{H}$ is determined for each of the $L$ submodels according to

$$
\left(\underline{\mathbf{A}}_{\varsigma}-\mathbf{H C}\right) \cdot \hat{\mathbf{v}}_{\varsigma}+\underline{\mathbf{B}}_{\varsigma} \cdot \mathbf{u}+\mathbf{H} \cdot \underline{\mathbf{y}}_{\mathrm{m}}=\dot{\hat{\mathbf{v}}}_{\varsigma} \leq \dot{\hat{\mathbf{x}}}
$$

and

$$
\dot{\hat{\mathbf{x}}} \leq \dot{\hat{\mathbf{w}}}_{\varsigma}=\left(\overline{\mathbf{A}}_{\varsigma}-\mathbf{H C}\right) \cdot \hat{\mathbf{w}}_{\varsigma}+\overline{\mathbf{B}}_{\varsigma} \cdot \mathbf{u}+\mathbf{H} \cdot \overline{\mathbf{y}}_{\mathrm{m}} .
$$

The main advantage of this bank of parallel observers is not only the fact that the bounds of the computed state estimates become tighter due to a better representation of the physical couplings between the vector components of $\mathbf{p}$ (cf. (4)). In addition, it also offers the possibility to stabilize and optimize the error dynamics with gains of smaller absolute values. Reduced observer gains typically lead to smaller amplifications of noise and therefore help to reduce the influence of the errors $\boldsymbol{\zeta}$ in a more reliable way, cf. (15). In such a way they also help to reduce the specified $H_{\infty}$ norm in the observer design.

Figs. 2 and 3 provide a representative comparison between the lower and upper state bounds for the case of evaluating the system dynamics in a pure open-loop fashion (result of the offline identification) and for the optimized observer parameterization O1. For the application at hand, it turned out that the sparse solutions $\mathbf{O} 2$ and $\mathbf{O} 3$ lead to practically identical results as the computationally more complex observer gain from $\mathbf{O 1}$, where the optimal non-sparse structure was determined by means of an LMI-constrained optimization, cf. [45]. Information about the offline design complexity for the observer design is summarized in Tab. 1. 
Table 1: Computing times and dimensions of the optimization tasks O1, O2, and O3 per iteration on an Intel XEON E5-2609v2, 2.5 GHz, 64 GB RAM, Windows 10, MATLAB R2018a, SeDuMi 1.3 [51]

\begin{tabular}{l|l|l} 
Scenario & $\begin{array}{l}\text { upper bound for the } \\
\text { computing time }\end{array}$ & $\begin{array}{l}\text { number of decision } \\
\text { variables }\end{array}$ \\
\hline \hline O1 & $1800 \mathrm{~s}$ & 446 \\
\hline O2 & $450 \mathrm{~s}$ & 307 \\
\hline O3 & $450 \mathrm{~s}$ & 302 \\
\hline
\end{tabular}

Table 2: Comparison of the offline identification with optimized observers

\begin{tabular}{|c|c|c|}
\hline Scenario & $\begin{array}{l}\text { Average diameter of rod } \\
\text { without additional noise }\end{array}$ & $\begin{array}{l}\text { emperature intervals } \\
\text { with additional noise }\end{array}$ \\
\hline$\overline{\mathbf{H}}=\mathbf{0}, 15 \cdot 10^{4}$ subdiv. & $6.4810 \mathrm{~K}$ & - \\
\hline $\mathbf{H}=\mathbf{0}, 10 \cdot 10^{4}$ subdiv. & $6.8631 \mathrm{~K}$ & - \\
\hline $\mathbf{H}=\mathbf{0}$, after merging & $23.4669 \mathrm{~K}$ & - \\
\hline $\mathbf{H}=\kappa \cdot \mathbf{C}^{T}, \kappa=1$ & $4.0271 \mathrm{~K}$ & $4.4533 \mathrm{~K}$ \\
\hline $\mathbf{H}=\kappa \cdot \mathbf{C}^{T}, \kappa=10$ & $3.8713 \mathrm{~K}$ & $4.4098 \mathrm{~K}$ \\
\hline $\mathbf{H}=\kappa \cdot \mathbf{C}^{T}, \kappa=50$ & $3.8536 \mathrm{~K}$ & $4.3974 \mathrm{~K}$ \\
\hline O1 & $3.9046 \mathrm{~K}$ & $4.4304 \mathrm{~K}$ \\
\hline O2 avg. gain: 4.2830 & $3.8984 \mathrm{~K}$ & $4.4245 \mathrm{~K}$ \\
\hline O3 avg. gain: 5.6930 & $3.8869 \mathrm{~K}$ & $4.4197 \mathrm{~K}$ \\
\hline
\end{tabular}

In Tab. 2, the width of the computed state enclosures - after subdivision of the parameter domain and elimination of inconsistent values during the identification stage - but without measurement feedback (i.e., for the case $\mathbf{H}=\mathbf{0}$ ) has been compared for three different scenarios. Firstly, the enclosures were evaluated for $15 \cdot 10^{4}$ subdivisions of the original parameter domain, leading to 39528 interval boxes covering approx. $0.32 \%$ of the original parameter domain (see case 3 in [44]). Secondly, the case of $10 \cdot 10^{4}$ subdivisions was investigated with 28302 resulting interval boxes (approx. $0.38 \%$ of the original parameter domain). Re-approximating the domain after $10 \cdot 10^{4}$ subdivisions by the routine [22] into pair-wise non-overlapping boxes leads to a list of $L=362$ intervals, where the lower and upper bounding systems corresponding to the matrices $\underline{\mathbf{A}}_{\varsigma}$ and $\overline{\mathbf{A}}_{\varsigma}, \varsigma \in\{1, \ldots, L\}$, are each Metzler and Hurwitz.

For all following simulations, as well as for all presented experimental results, the sampling time for the evaluation of the state equations by means of the solver ode3 in SimulinK is set to the sensors' sampling time of $0.1 \mathrm{~s}$.

As it can be seen from the comparison of the pure open-loop simulation re- 


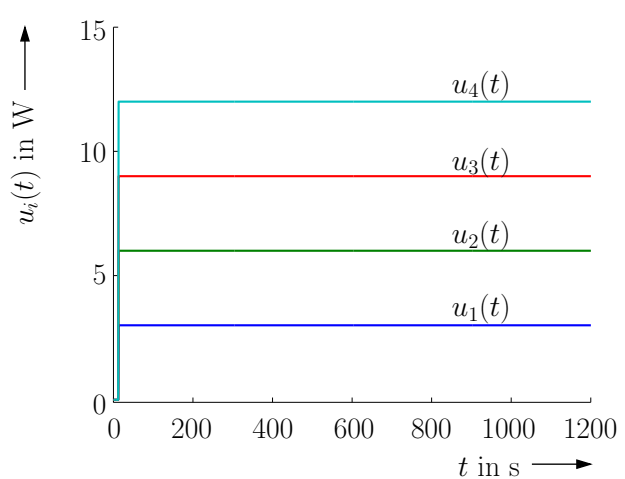

(a) Control signals $\mathbf{u}(t)$.

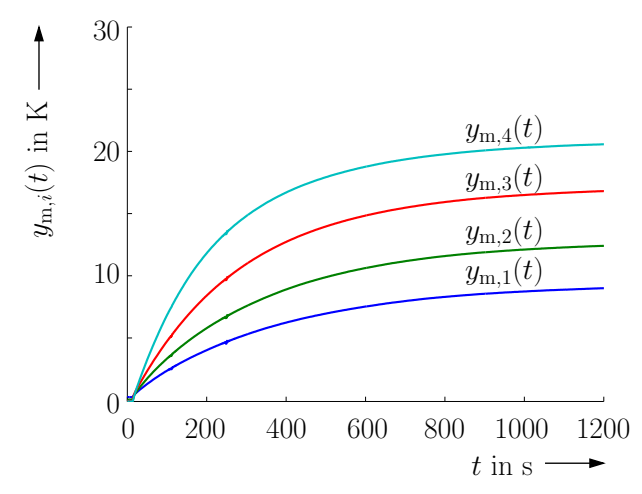

(b) Measured rod temperatures.

Figure 2: Inputs and measurements in the experiment.

sults in terms of the average interval diameters over the time horizon of $1200 \mathrm{~s}$, the replacement by a smaller number of boxes leads to significantly wider interval diameters if measurement information is not accounted for. This widening of the computed interval bounds results from a less accurate representation of the mutual dependence in the uncertainty between the individual parameters. Exemplarily, this dependence is shown in Fig. 4a for the relation between $\alpha_{\mathrm{B}}, \alpha_{\mathrm{T}}$, and $\Delta \alpha$ as well as for $\alpha_{\mathrm{B}}, \alpha_{\mathrm{T}}$, and $\lambda_{\mathrm{R}}$ in Fig. 4b. However, a reduction of the number of boxes is inevitable to obtain system models that can be evaluated in real time by the bank of interval observers. Moreover, it has to be noted that a naive replacement of all resulting boxes (either after $10 \cdot 10^{4}$ or $15 \cdot 10^{4}$ subdivisions) is not feasible due to the fact that the upper bounding matrix turns into an unstable model due to the mutual couplings between both vectors $\mathbf{p}$ and $\overline{\mathbf{p}}$. Such a coarse enclosure is also not further investigated because it can be shown that the resulting error dynamics cannot be stabilized with the help of the structurally predefined sparse observer gain matrix according to (11).

For the optimization of the observer bank with $L=362$ parameter boxes, the outcomes for the options $\mathbf{O 1}, \mathbf{O 2}$, and $\mathbf{O 3}$ are not only compared for the raw measured data but also with measurements artificially corrupted by measurement noise according to the bounded tolerance of $\pm 0.75 \mathrm{~K}$. The comparison shows the excellent attenuation of noise and moreover highlights the fact that for the application at hand all three options provide quite similar estimation results. Now, these results are compared with naive trial-and-error parameterizations of (11) by successively increasing the gain from $\kappa=1$ to $\kappa=50$. With respect to the widths of the estimation results, the naive choices $\kappa=10$ and $\kappa=50$ are slightly better than the optimized results in a noise-free setting but not in the practically relevant case in which disturbances are present inevitably. Note that this noise-free setting is not a fully fair comparison since the observer parameterization does not directly minimize this average diameter of the state estimates but aims at reducing the 


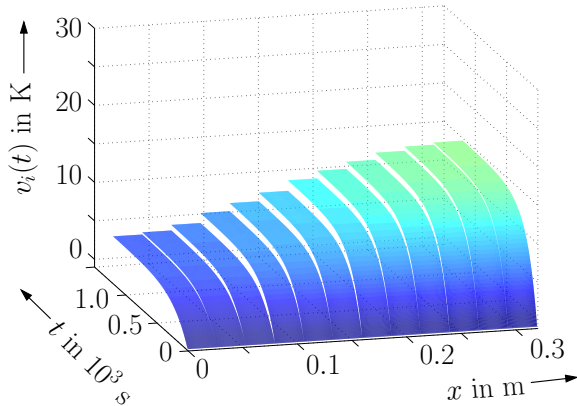

(a) Lower bounds $v_{i}(t)$.

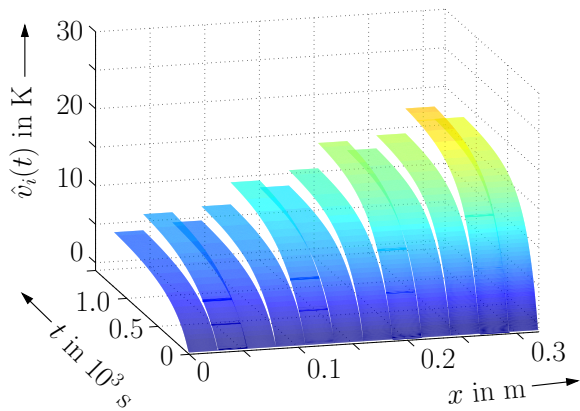

(c) Lower bounds $\hat{v}_{i}(t)$, case $\mathbf{O} \mathbf{1}$.

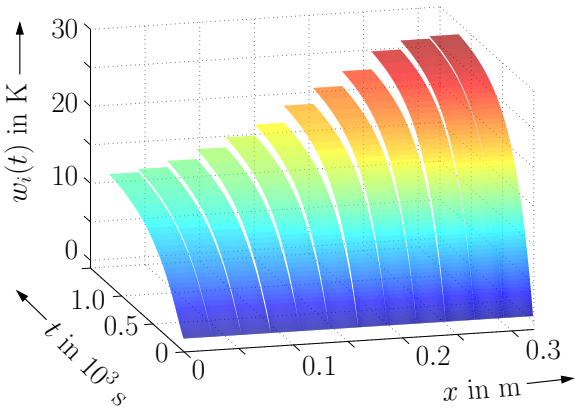

(b) Upper bounds $w_{i}(t)$.

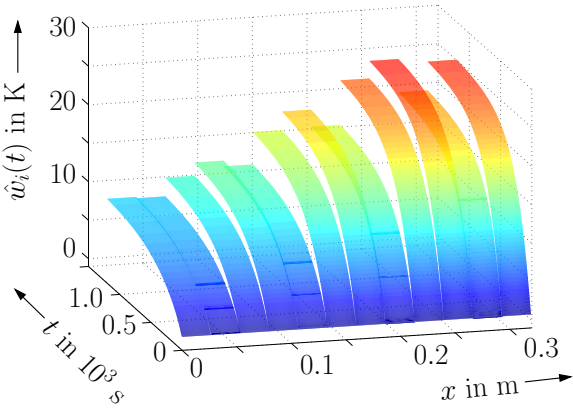

(d) Upper bounds $\hat{w}_{i}(t)$, case $\mathbf{O} 1$.

Figure 3: Bounds for the rod temperatures in the offline identification (Figs. 3a, 3b) as well as in the online state observation (Figs. 3c, 3d).

sensitivity of the estimated state trajectories by the employed $H_{\infty}$ design. However, it can be shown that the proposed optimization procedure directly produces optimal parameterizations without the need for any experimental parameter tuning. Moreover, the options $\mathbf{O 2}$ and $\mathbf{O 3}$ lead to smaller average gain values than $\kappa=10$ and, hence, prevent the use of excessively large gains ${ }^{6}$. Such large gains may not only lead to the amplification of measurement noise in terms of a deterioration of the estimated interval bounds but may also produce unnecessarily stiff observer dynamics. Therefore, it can be observed that the suggested optimization routine directly obeys the two fundamental rules of control and estimator design, namely, stability and the preservation of the "natural" dynamics in the sense that physically slow systems should remain slow and that fast systems are not forced

\footnotetext{
${ }^{6}$ Note, using a larger number of decision variables, e.g. by employing $\mathbf{O 2}$ instead of $\mathbf{O 3}$ does not necessarily lead to tighter interval bounds but — due to the employed performance indicators it inevitably leads to smaller gains achieving similar estimation results with a reduced sensitivity against noise and model inaccuracies.
} 


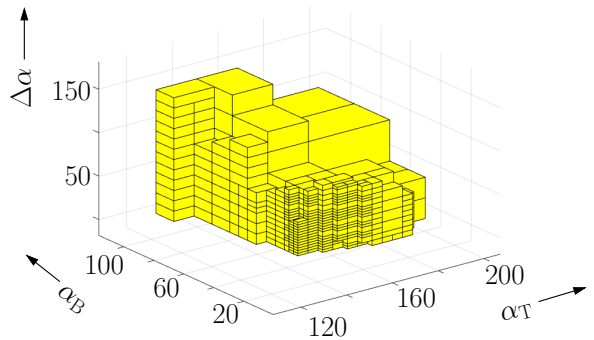

(a) Identification of $\Delta \alpha$ in dependence of $\alpha_{\mathrm{B}}$ and $\alpha_{\mathrm{T}}$.

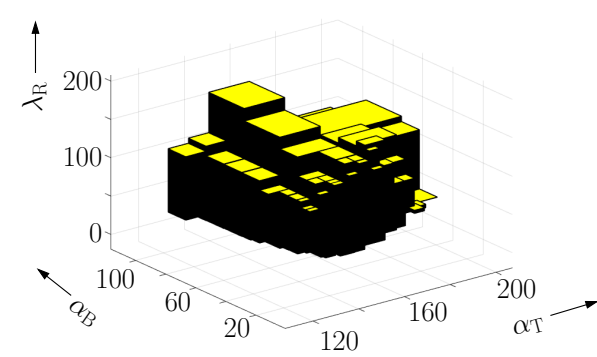

(b) Identification of $\lambda_{\mathrm{R}}$ in dependence of $\alpha_{\mathrm{B}}$ and $\alpha_{\mathrm{T}}$.

Figure 4: Representative result of the verified parameter identification.

to become unnecessarily slow, where the violation of the latter design rule would typically lead to large gains and increased sensitivity against disturbances (as well as large control amplitudes).

Future work can deal with procedures allowing for a (partial) decoupling of the entries in the parameter vector $\mathbf{p}$ by a linear change of coordinates. However, the drawback of such a change of coordinates is the loss of information about natural range constraints which could be obtained again by combinations with the contractor-based identification routine presented in [47].

\section{Cooperativity-Preserving Closed-Loop Control}

\subsection{Naive Implementation}

For the development of a first, naive implementation of a cooperativity-preserving feedback controller, assume that all system states $\mathbf{x}(t)$ can be measured or estimated perfectly (without the interval tolerances described before). Then, the input signal

$$
\mathbf{u}=\mathbf{u}_{\mathrm{ff}}-\mathbf{K} \cdot \mathbf{x}
$$

with the gain matrix $\mathbf{K}$ and the feedforward control signal $\mathbf{u}_{\mathrm{ff}}(t)$ represents a classical state feedback control approach. Parameterizing this controller by

$$
\mathbf{K}=\nu \cdot\left[\begin{array}{c}
\mathbf{I}_{N \times N} \otimes \mathbf{1}_{2 M+1} \\
\mathbf{0}_{\tilde{N} \times N}
\end{array}\right]^{T}
$$


for the case $M=0$ (representing a finite volume discretization of the rod in Fig. 1 into $\tilde{N}=4$ elements) as well as

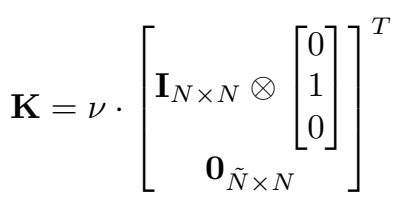

for $M=1$ (leading to $\tilde{N}=12$ segments) can be used to ensure asymptotic stability of the closed-loop dynamics and cooperativity of the closed control loop [44].

Obviously, this approach is valid for semi-discretizations with $M=0$ and $M=1$ because the resulting controller does not modify any of the entries in the system matrix $\mathbf{A}(\mathbf{p})$ which are zero in the open-loop case. For non-negative gain values with $\nu \geq 0$, asymptotically stabilizing solutions can be found if the open-loop model A (p) itself (respectively, the corresponding lower and upper bounding matrices) are asymptotically stable. Applying the Gershgorin circle theorem to the closed-loop differential equations, it can be shown that (for a system model without parameter uncertainty), the closed-loop controller is guaranteed to be asymptotically stable for all $0<\nu<\nu^{*}$ satisfying the inequalities

$$
\frac{1}{c_{i} \cdot m_{i}} \cdot\left(p_{1}+p_{2}+p_{4}\right)-q=-\frac{\alpha_{\mathrm{B}} \cdot A_{\mathrm{s}}}{c_{i} \cdot m_{i}}-q<0
$$

for $i \in\{1, \tilde{N}\}$ as well as

$$
\frac{1}{c_{i} \cdot m_{i}} \cdot\left(p_{3}+2 p_{2}+p_{4}\right)-q=-\frac{\alpha_{\mathrm{B}} \cdot A_{\mathrm{s}}}{c_{i} \cdot m_{i}}-q<0
$$

for $i \in\{2, \ldots, \tilde{N}-1\}$ with

$$
q:=\frac{\nu}{c_{i} \cdot m_{i}} \cdot \frac{1}{2 M+1} \geq 0 .
$$

In addition, the Metzler structure of the open-loop system matrix is preserved if all off-diagonal elements of $\mathbf{A}(\mathbf{p})-\mathbf{B} \cdot \mathbf{K}$ of the closed-loop system remain non-negative according to

$$
\frac{p_{2}}{c_{i} \cdot m_{i}}-q \geq 0 .
$$

For the system under consideration, this leads to the constraint

$$
\nu<\nu^{*}=(2 M+1) \cdot p_{2} .
$$

Taking into account the parameter values identified in [44], after $15 \cdot 10^{4}$ interval subdivisions, the upper bound $\nu^{*}=0.7131$ is obtained. This value reduces to $\nu^{*}=0.6803$ for the replacement of the list of originally 39528 possibly feasible 
boxes by the outer hull represented by 362 intervals which was also used in the previous section for the design of a bank of parallel observers ${ }^{7}$.

Note that this naive control approach is not feasible (due to violation of the Metzler constraint) if discretizations with $M \geq 2$ are used. Moreover, optimality of this controller with respect to a minimum diameter of the resulting state bounds as well as insensitivity with respect to non-modeled disturbances and noise in state measurement and reconstruction are not ensured by this approach. Therefore, the following subsection deals with abolishing the structural constraints given in Eqs. (33) and (34) by optimizing the controller gains in combination with a cooperative interval observer by means of an LMI-constrained design procedure.

\subsection{Optimized Implementation}

For the optimization of cooperativity-preserving feedback controllers, the duality to the design of a bank of parallel interval observers presented in Sec. 2.4 is employed. For that purpose, independent gains $\underline{\mathbf{K}}_{\varsigma}$ and $\overline{\mathbf{K}}_{\varsigma}$ are defined for the lower and upper state estimates determined by each of the $L$ observers and optimized in the feedback controller

$$
\mathbf{u}=\mathbf{u}_{\mathrm{ff}}-\sum_{\varsigma=1}^{L}\left(\underline{\mathbf{K}}_{\varsigma} \hat{\mathbf{v}}_{\varsigma}+\overline{\mathbf{K}}_{\varsigma} \hat{\mathbf{w}}_{\varsigma}\right)=\mathbf{u}_{\mathrm{ff}}-\boldsymbol{\nu} .
$$

In analogy to the previous subsection where a naive control structure was considered, the feedforward signal $\mathbf{u}_{\mathrm{ff}}(t)$ in (38) represents a further degree of freedom that can be chosen such that tracking control properties are enhanced and overshooting output limits is reduced as far as possible.

However, the main goals of the control design are the reduction of the interval widths of the resulting bounds of reachable states, the reduction of sensitivity against external disturbances, and the possibility to find a trade-off between control accuracy and the required amplitudes of the system's input signals. To be able to predict the closed-loop output trajectories in the same manner as for the previous observer parameterization, it is desired additionally to ensure cooperativity for the complete system structure after introduction of the controller (38). To achieve these goals, the following (auxiliary) state vectors are defined and included in the offline control parameterization [46]:

- Worst-case lower bounds $\underline{\mathbf{x}}(t)$ of the true, however, unknown states (determined, for example, by a pure evaluation of the open-loop ODEs without observer-based measurement feedback);

- Worst-case upper bounds $\overline{\mathbf{x}}(t)$ of the true, however, unknown states;

- The worst-case, component-wise non-negative deviation

$$
\boldsymbol{\mu}_{i}=\hat{\mathbf{v}}_{i}-\underline{\mathbf{x}}
$$

between the lower state estimates $\hat{\mathbf{v}}_{i}(t)$ of the $i$-th observer and $\underline{\mathbf{x}}(t)$;

\footnotetext{
${ }^{7}$ For the uncertain model with the lower and upper bounds (5) of the system matrix, $\mathbf{A}(\underline{\mathbf{p}})-$ $\mathbf{B} \cdot \mathbf{K}$ is typically most critical with respect to a violation of the Metzler property for too large values $\nu$, while $\mathbf{A}(\overline{\mathbf{p}})-\mathbf{B} \cdot \mathbf{K}$ usually represents the subsystem exhibiting instability.
} 
- The component-wise defined diameters

$$
\boldsymbol{\epsilon}_{i}=\hat{\mathbf{w}}_{i}-\hat{\mathbf{v}}_{i}
$$

of the state estimates provided by the $i$-th interval observer;

- The worst-case, component-wise non-negative deviation

$$
\boldsymbol{\eta}_{i}=\overline{\mathbf{x}}-\hat{\mathbf{w}}_{i}
$$

between the upper state estimates $\hat{\mathbf{w}}_{i}(t)$ of the $i$-th observer and $\overline{\mathbf{x}}(t)$.

To ensure cooperativity in addition to the stability and insensitivity properties mentioned above, the joint set of ODEs

$$
\frac{\mathrm{d}}{\mathrm{d} t}\left[\begin{array}{c}
\underline{\mathbf{x}} \\
\boldsymbol{\mu}_{1} \\
\vdots \\
\boldsymbol{\mu}_{L} \\
\boldsymbol{\epsilon}_{1} \\
\vdots \\
\boldsymbol{\epsilon}_{L} \\
\overline{\mathbf{x}}
\end{array}\right]=\underbrace{\left(\left[\begin{array}{cc}
\mathbb{A}_{1,1} & \mathbf{0} \\
\mathbf{0} & \mathbb{A}_{2,2}
\end{array}\right]-\left[\begin{array}{cc}
\mathbb{B}_{1,1} & \mathbf{0} \\
\mathbf{0} & \mathbb{B}_{2,2}
\end{array}\right] \cdot\left[\begin{array}{cc}
\mathbb{K}_{1,1} & \mathbf{0} \\
\mathbf{0} & \mathbb{K}_{2,2}
\end{array}\right]\right)}_{=\mathbb{A}_{\mathrm{K}}\left(\underline{\mathbf{K}}_{\varsigma}, \overline{\mathbf{K}}_{\varsigma}\right)} \cdot\left[\begin{array}{c}
\underline{\mathbf{x}} \\
\boldsymbol{\mu}_{1} \\
\vdots \\
\boldsymbol{\mu}_{L} \\
\boldsymbol{\epsilon}_{1} \\
\vdots \\
\boldsymbol{\epsilon}_{L} \\
\overline{\mathbf{x}}(t)
\end{array}\right]+\tilde{\mathbb{S}} \cdot\left[\begin{array}{c}
\mathbf{y}_{\mathrm{d}} \\
\mathbf{y}_{\mathrm{m}}
\end{array}\right]
$$

comprising the dynamics of the closed-loop control system and all $L$ interval-based state observers is taken into consideration. Here, the ODEs are expressed in terms of the auxiliary states (39) and (40). Differentiating their definitions with respect to time and inserting the corresponding ODEs for both, the closed-loop control structure and the respective observers, the ODEs (42) consist of the matrix blocks

$$
\begin{aligned}
& \mathbb{A}_{1,1}=\left[\begin{array}{ccccccc}
\underline{\mathbf{A}} & \mathbf{0} & \mathbf{0} & \ldots & \mathbf{0} & \mathbf{0} & \ldots \\
\underline{\mathbf{A}}_{1}-\mathbf{H} \mathbf{C}-\underline{\mathbf{A}} & \underline{\mathbf{A}}_{1}-\mathbf{H C} & \mathbf{0} & \ldots & \mathbf{0} & \mathbf{0} & \ldots \\
\underline{\mathbf{A}}_{2}-\mathbf{H C}-\underline{\mathbf{A}} & \mathbf{0} & \underline{\mathbf{A}}_{2}-\mathbf{H C} & \ldots & \mathbf{0} & \mathbf{0} & \ldots \\
\vdots & & & & & & \\
\mathbf{0} & \overline{\mathbf{A}}_{1}-\underline{\mathbf{A}}_{1} & \mathbf{0} & \ldots & \overline{\mathbf{A}}_{1}-\underline{\mathbf{A}}_{1}-\mathbf{H C} & \mathbf{0} & \ldots \\
\mathbf{0} & \mathbf{0} & \overline{\mathbf{A}}_{2}-\underline{\mathbf{A}}_{2} & \ldots & \mathbf{0} & \overline{\mathbf{A}}_{2}-\underline{\mathbf{A}}_{2}-\mathbf{H C} & \ldots \\
\vdots & & & & & &
\end{array}\right] \\
& =\mathbf{J}_{1,1} \otimes \underline{\mathbf{A}}+\left(\sum_{\varsigma=1}^{L}-\mathbf{J}_{\varsigma+1,1} \otimes \underline{\mathbf{A}}+\left(\mathbf{J}_{\varsigma+1,1}+\mathbf{J}_{\varsigma+1, \varsigma+1}\right) \otimes\left(\underline{\mathbf{A}}_{\varsigma}-\mathbf{H C}\right)\right. \\
& +\left(\mathbf{J}_{L+\varsigma+1, \varsigma+1}+\mathbf{J}_{L+\varsigma+1, L+\varsigma+1}\right) \otimes\left(\overline{\mathbf{A}}_{\varsigma}-\underline{\mathbf{A}}_{\varsigma}\right) \\
& \left.-\mathbf{J}_{L+\varsigma+1, L+\varsigma+1} \otimes(\mathbf{H C})\right) \text {, }
\end{aligned}
$$




$$
\begin{aligned}
& \mathbb{B}_{1,1}=\left[\begin{array}{ll}
\mathbf{1}_{(2 L+1)}^{T} & 0
\end{array}\right] \otimes\left[\begin{array}{c}
\underline{\mathbf{B}} \\
\underline{\mathbf{B}}_{1}-\underline{\mathbf{B}} \\
\underline{\mathbf{B}}_{2}-\underline{\mathbf{B}} \\
\vdots \\
\overline{\mathbf{B}}_{1}-\underline{\mathbf{B}}_{1} \\
\overline{\mathbf{B}}_{2}-\underline{\mathbf{B}}_{2} \\
\vdots
\end{array}\right], \\
& \mathbb{B}_{2,2}=\overline{\mathbf{B}}, \\
& \mathbb{K}_{1,1}=\sum_{\varsigma=1}^{L}\left(\left(\mathbf{J}_{\varsigma, 1}+\mathbf{J}_{\varsigma, \varsigma+1}\right) \otimes \underline{\mathbf{K}}_{\varsigma}+\left(\mathbf{J}_{L+\varsigma, 1}+\mathbf{J}_{L+\varsigma, \varsigma+1}+\mathbf{J}_{L+\varsigma, L+1+\varsigma}\right) \otimes \overline{\mathbf{K}}_{\varsigma}\right)
\end{aligned}
$$

as well as

$$
\mathbb{K}_{2,2}=\sum_{\varsigma=1}^{L}\left(\underline{\mathbf{K}}_{\varsigma}+\overline{\mathbf{K}}_{\varsigma}\right) .
$$

In the equations above, $\mathbf{1}_{(2 L+1)} \in \mathbb{R}^{(2 L+1)}$ represents a column vector containing the value 1 in all entries and $\mathbf{J}_{\mu, \nu} \in \mathbb{R}^{(2 L+1) \times(2 L+1)}$ is a matrix defined according to

$$
\mathbf{J}_{\mu, \nu}=\left(J_{i, j}\right)= \begin{cases}J_{i, j}=1 & \text { for } \quad(i, j)=(\mu, \nu) \\ J_{i, j}=0 & \text { else }\end{cases}
$$

Moreover, the term $\tilde{\mathbb{S}} \cdot\left[\begin{array}{ll}\mathbf{y}_{\mathrm{d}}^{T} & \mathbf{y}_{\mathrm{m}}^{T}\end{array}\right]^{T}$ in (42) characterizes the influence of the vector-valued reference signal in the multi-input multi-output control system as well as the influence of the measurement feedback in the observers' ODEs. This term is not specified further in this section because it does not have any influence on the optimization of the required controller gains. Additional details about the control structure and a full derivation of the equations above can be found in [46].

The control synthesis follows the duality principle in comparison to the previous observer design by specifying an $H_{\infty}$ design task for the output vector

$$
\begin{gathered}
\mathbf{y}_{\infty}=\mathcal{C}_{\infty} \cdot \boldsymbol{\zeta}+\mathcal{D}_{\infty 2} \cdot \boldsymbol{\nu}+\mathcal{D}_{\infty 1} \cdot \mathbf{w}, \quad \boldsymbol{\zeta}=\left[\frac{\mathbf{x}}{\overline{\mathbf{x}}}\right] \\
\mathcal{C}_{\infty}=\left[\begin{array}{cc}
-\boldsymbol{\Xi} \mathbf{I}_{n_{\mathrm{x}} \times n_{\mathrm{x}}} & \boldsymbol{\Xi} \mathbf{I}_{n_{\mathrm{x}} \times n_{\mathrm{x}}} \\
\mathbf{0} & \mathbf{0} \\
\mathbf{0} & \mathbf{0}
\end{array}\right], \mathcal{D}_{\infty 1}=\left[\begin{array}{c}
\mathbf{0} \\
L \mathbf{I}_{n_{\nu} \times n_{\nu}} \\
\mathbf{0}
\end{array}\right], \mathcal{D}_{\infty 2}=\left[\begin{array}{c}
\mathbf{0} \\
\mathbf{0} \\
\boldsymbol{\Gamma \mathbf { I } _ { n _ { \nu } \times n _ { \nu } }}
\end{array}\right] .
\end{gathered}
$$

In (50), the individual terms (listed in the order from left to right) correspond to

C1 weighting the difference between the guaranteed lower and upper state bounds $\underline{\mathbf{x}}(t)$ and $\overline{\mathbf{x}}(t)$ of the closed-loop dynamics by the factors $\xi_{j}>0, \boldsymbol{\Xi}=\operatorname{diag}\left\{\xi_{j}\right\}$, in comparison to

C2 the control effort (penalized by the factors $\gamma_{j}>0, \boldsymbol{\Gamma}=\operatorname{diag}\left\{\gamma_{j}\right\}$ ) and 
C3 the disturbance inputs $\mathbf{w}(t)$.

To fulfill all criteria mentioned above, the property $\mathbf{C} \mathbf{1}$ is refined by accounting for the augmented system model (42) in terms of the criteria:

C1a Maximize the lower bounds of all state observers by maximizing the distances $\boldsymbol{\mu}_{i}(t)$

C1b Minimize the distances $\boldsymbol{\epsilon}_{i}(t)$ between the lower and upper state estimates;

C1c Minimize the distance $\overline{\mathbf{x}}(t)-\underline{\mathbf{x}}(t)$.

Using the linearizing change of variables

$$
\mathbf{Q}=\mathbf{P}^{-1} \succ 0 \text { as well as } \underline{\mathbf{Y}}_{\varsigma}=\underline{\mathbf{K}}_{\varsigma} \mathbf{Q} \text { and } \quad \overline{\mathbf{Y}}_{\varsigma}=\overline{\mathbf{K}}_{\varsigma} \mathbf{Q}
$$

for an $H_{\infty}$ control design in combination with a quadratic Lyapunov function candidate (specified by $\mathbf{P}=\mathbf{P}^{T} \succ 0$ ), the system matrix $\mathbb{A}_{K}\left(\underline{\mathbf{K}}_{\varsigma}, \overline{\mathbf{K}}_{\varsigma}\right)$ in (42) needs to be multiplied from the right-hand side in a block-wise manner with the positive definite matrix $\mathbf{Q}$. In terms of the Kronecker product $\otimes$, this leads to

$$
\mathbb{A}_{\mathrm{Q}}=\left(\left[\begin{array}{cc}
\mathbb{A}_{1,1} & \mathbf{0} \\
\mathbf{0} & \mathbb{A}_{2,2}
\end{array}\right]-\left[\begin{array}{cc}
\mathbb{B}_{1,1} & \mathbf{0} \\
\mathbf{0} & \mathbb{B}_{2,2}
\end{array}\right] \cdot\left[\begin{array}{cc}
\mathbb{K}_{1,1} & \mathbf{0} \\
\mathbf{0} & \mathbb{K}_{2,2}
\end{array}\right]\right) \cdot\left(\mathbf{I}_{(2 L+2) \times(2 L+2)} \otimes \mathbf{Q}\right)
$$

which is identical to

$$
\mathbb{A}_{\mathbf{Q}}=\left[\begin{array}{cc}
\mathbb{A}_{1,1} \cdot\left(\mathbf{I}_{(2 L+1) \times(2 L+1)} \otimes \mathbf{Q}\right) & \mathbf{0} \\
\mathbf{0} & \mathbb{A}_{2,2} \cdot \mathbf{Q}
\end{array}\right]-\left[\begin{array}{cc}
\mathbb{B}_{1,1} \cdot \mathbb{Y}_{1,1} & \mathbf{0} \\
\mathbf{0} & \mathbb{B}_{2,2} \cdot \mathbb{Y}_{2,2}
\end{array}\right]
$$

with

$$
\mathbb{Y}_{1,1}=\sum_{\varsigma=1}^{L}\left(\left(\mathbf{J}_{\varsigma, 1}+\mathbf{J}_{\varsigma, \varsigma+1}\right) \otimes \underline{\mathbf{Y}}_{\varsigma}+\left(\mathbf{J}_{L+\varsigma, 1}+\mathbf{J}_{L+\varsigma, \varsigma+1}+\mathbf{J}_{L+\varsigma, L+1+\varsigma}\right) \otimes \overline{\mathbf{Y}}_{\varsigma}\right)
$$

and

$$
\mathbb{Y}_{2,2}=\sum_{\varsigma=1}^{L}\left(\underline{\mathbf{Y}}_{\varsigma}+\overline{\mathbf{Y}}_{\varsigma}\right)
$$

where the matrices $\underline{\mathbf{Y}}_{\varsigma}$ and $\overline{\mathbf{Y}}_{\varsigma}$ were defined in (52).

\subsection{Simplified Control Design for Two Bounding Systems}

If only two bounding systems $\underline{\mathbf{A}}$ and $\overline{\mathbf{A}}$ (corresponding to the first and last vector rows in (42)) with the matrices $\boldsymbol{B}_{1}$ of feedforward and disturbance inputs as well as $\boldsymbol{\mathcal { B }}_{2}$ for the feedback control inputs were accounted for according to

$$
\mathcal{A}=\left[\begin{array}{ll}
\frac{\mathbf{A}}{\mathbf{0}} & \frac{\mathbf{0}}{\mathbf{A}}
\end{array}\right], \quad \mathcal{B}_{1}=L \cdot\left[\frac{\overline{\mathbf{B}}}{\overline{\mathbf{B}}}\right], \quad \mathcal{B}_{2}=\left[\frac{\overline{\mathbf{B}}}{\overline{\mathbf{B}}}\right],
$$


the design LMIs $\quad\left[\begin{array}{ccc}\mathcal{S}_{1,1} & \mathcal{B}_{1} & \mathcal{S}_{1,3} \\ \mathcal{B}_{1}^{T} & -\gamma_{\infty} \mathbf{I} & \mathcal{D}_{\infty 1}^{T} \\ \mathcal{S}_{3,1} & \mathcal{D}_{\infty 1} & -\gamma_{\infty} \mathbf{I}\end{array}\right] \prec 0$

with

$$
\begin{aligned}
\mathcal{S}_{1,1}= & \left(\mathbf{I}_{2 \times 2} \otimes \mathbf{Q}\right) \mathcal{A}^{T}-\left[\sum_{\varsigma=1}^{L} \underline{\mathbf{Y}}_{\varsigma} \sum_{\varsigma=1}^{L} \overline{\mathbf{Y}}_{\varsigma}\right]^{T} \cdot \mathcal{B}_{2}^{T} \\
& +\underbrace{\mathcal{A}\left(\mathbf{I}_{2 \times 2} \otimes \mathbf{Q}\right)-\mathcal{B}_{2} \cdot\left[\sum_{\varsigma=1}^{L} \underline{\mathbf{Y}}_{\varsigma} \sum_{\varsigma=1}^{L} \overline{\mathbf{Y}}_{\varsigma}\right]}_{\mathcal{A}_{\mathrm{Q}}}
\end{aligned}
$$

and

$$
\mathcal{S}_{1,3}=\mathcal{S}_{3,1}^{T}=\left(\mathbf{I}_{2 \times 2} \otimes \mathbf{Q}\right) \mathcal{C}_{\infty}^{T}-\left[\sum_{\varsigma=1}^{L} \underline{\mathbf{Y}}_{\varsigma} \sum_{\varsigma=1}^{L} \overline{\mathbf{Y}}_{\varsigma}\right]^{T} \cdot \mathcal{D}_{\infty 2}^{T}
$$

would be obtained, where the parameter $\gamma_{\infty}>0$ needs to be minimized to achieve optimality.

\subsection{Input-to-State Stability and Cooperativity of the Com- plete List of Bounding Systems}

ISS of each of the $L$ bounding systems with $\mathbf{A} \in\left[\underline{\mathbf{A}}_{i} ; \overline{\mathbf{A}}_{i}\right]$ and $\mathbf{B} \in\left[\underline{\mathbf{B}}_{i} ; \overline{\mathbf{B}}_{i}\right]$ is guaranteed for each possible $i \in\{1, \ldots, L\}$ if the LMIs

$$
\begin{aligned}
& \left(\mathbf{I}_{2 \times 2} \otimes \mathbf{Q}\right)\left[\begin{array}{cc}
\underline{\mathbf{A}}_{i} & \frac{\mathbf{0}}{\mathbf{0}}
\end{array}\right]^{T}-\left[\begin{array}{ll}
\sum_{\varsigma=1}^{L} \underline{\mathbf{Y}}_{\varsigma} & \sum_{\varsigma=1}^{L} \overline{\mathbf{Y}}_{\varsigma}
\end{array}\right]^{T} \cdot \mathcal{B}_{2, i}^{T} \\
& \quad+\left[\begin{array}{cc}
\underline{\mathbf{A}}_{i} & \mathbf{0} \\
\mathbf{0} & \overline{\mathbf{A}}_{i}
\end{array}\right]\left(\mathbf{I}_{2 \times 2} \otimes \mathbf{Q}\right)-\boldsymbol{B}_{2, i} \cdot\left[\begin{array}{ll}
\sum_{\varsigma=1}^{L} \underline{\mathbf{Y}}_{\varsigma} & \left.\sum_{\varsigma=1}^{L} \overline{\mathbf{Y}}_{\varsigma}\right] \prec 0, \quad \mathcal{B}_{2, i}=\left[{\underline{\mathbf{B}}}_{i}\right. \\
\overline{\mathbf{B}}_{i}
\end{array}\right]
\end{aligned}
$$

are feasible. In addition to the stability requirement (61), the gains $\underline{\mathbf{K}}_{\varsigma}$ and $\overline{\mathbf{K}}_{\varsigma}$ should be chosen in such a way that the matrices

$$
\begin{aligned}
& \mathcal{M}_{0}\left(\underline{\mathbf{K}}_{\varsigma}, \overline{\mathbf{K}}_{\varsigma}\right)=\mathcal{A}-\mathcal{B}_{2} \cdot \sum_{\varsigma=1}^{L}\left(\underline{\mathbf{K}}_{\varsigma}+\overline{\mathbf{K}}_{\varsigma}\right) \quad \text { and } \\
& \mathcal{M}_{i}\left(\underline{\mathbf{K}}_{\varsigma}, \overline{\mathbf{K}}_{\varsigma}\right)=\mathcal{A}_{\mathrm{H}, i}-\mathcal{B}_{2, i} \cdot \sum_{\varsigma=1}^{L}\left(\underline{\mathbf{K}}_{\varsigma}+\overline{\mathbf{K}}_{\varsigma}\right), \mathcal{A}_{\mathrm{H}, i}=\left[\begin{array}{cc}
\underline{\mathbf{A}}_{i}-\mathbf{H C} & \mathbf{0} \\
\mathbf{0} & \overline{\mathbf{A}}_{i}-\mathbf{H C}
\end{array}\right]
\end{aligned}
$$

fulfill the sufficient criterion for cooperativity in terms of Metzler matrices. According to the observer design, the element-wise defined inequality constraints

$$
\begin{aligned}
& \operatorname{col}\left(\left(\mathcal{M}_{0}\left(\underline{\mathbf{Y}}_{\varsigma} \cdot \check{\mathbf{Q}}, \overline{\mathbf{Y}}_{\varsigma} \cdot \check{\mathbf{Q}}\right)\right) \circ(\mathbf{E}-\mathbf{I})\right) \geq \mathbf{0} \\
& \text { and } \quad \operatorname{col}\left(\left(\mathcal{M}_{i}\left(\underline{\mathbf{Y}}_{\varsigma} \cdot \check{\mathbf{Q}}, \overline{\mathbf{Y}}_{\varsigma} \cdot \check{\mathbf{Q}}\right)\right) \circ(\mathbf{E}-\mathbf{I})\right) \geq \mathbf{0}, \quad i \in\{1, \ldots, L\},
\end{aligned}
$$

are obtained by inserting the definition (52) into (62) and (63). As for the observer synthesis, an iterative solution procedure is established in which $\check{\mathbf{Q}}$ denotes 
the matrix $\mathbf{Q}$ obtained in the previous iteration step. This iteration is continued until (64) and (65) are satisfied and a suitable (e.g. quadratic) norm

$$
\|\check{\mathbf{Q}}-\mathbf{Q}\| \leq \epsilon_{\mathrm{Q}}
$$

falls below some positive threshold value $\epsilon_{\mathrm{Q}}>0$. In (64) and (65), $\mathbf{E}$ is a matrix of appropriate dimension containing the constant value 1 in all of its entries.

For practical implementations, it is reasonable to be able to limit the controller gains to some predefined upper and lower values, as an heuristics for handling input constraints. Due to the iterative control parameterization resulting from the multiplicative couplings between the unknown matrices in (64) and (65), the gain limitations are as well expressed by the element-wise defined inequalities

and

$$
\begin{aligned}
& \operatorname{col}\left(\left[\sum_{\varsigma=1}^{L} \underline{\mathbf{Y}}_{\varsigma} \sum_{\varsigma=1}^{L} \overline{\mathbf{Y}}_{\varsigma}\right] \cdot\left(\mathbf{I}_{2 \times 2} \otimes \check{\mathbf{Q}}\right)\right) \leq k_{\max }>0 \\
& \operatorname{col}\left(\left[\sum_{\varsigma=1}^{L} \underline{\mathbf{Y}}_{\varsigma} \sum_{\varsigma=1}^{L} \overline{\mathbf{Y}}_{\varsigma}\right] \cdot\left(\mathbf{I}_{2 \times 2} \otimes \check{\mathbf{Q}}\right)\right) \geq-k_{\max } .
\end{aligned}
$$

If the inequalities above hold, the element-wise defined inequalities

$$
\begin{array}{r}
\underline{\mathbf{A}}-\mathbf{H C}-\underline{\mathbf{B}} \cdot \sum_{\varsigma=1}^{L}\left(\underline{\mathbf{K}}_{\varsigma}+\overline{\mathbf{K}}_{\varsigma}\right) \leq \underline{\mathbf{A}}_{i}-\mathbf{H C}-\underline{\mathbf{B}}_{i} \cdot \sum_{\varsigma=1}^{L}\left(\underline{\mathbf{K}}_{\varsigma}+\overline{\mathbf{K}}_{\varsigma}\right) \\
\text { and } \quad \overline{\mathbf{A}}-\mathbf{H C}-\overline{\mathbf{B}} \cdot \sum_{\varsigma=1}^{L}\left(\underline{\mathbf{K}}_{\varsigma}+\overline{\mathbf{K}}_{\varsigma}\right) \geq \overline{\mathbf{A}}_{i}-\mathbf{H C}-\overline{\mathbf{B}}_{i} \cdot \sum_{\varsigma=1}^{L}\left(\underline{\mathbf{K}}_{\varsigma}+\overline{\mathbf{K}}_{\varsigma}\right)
\end{array}
$$

are satisfied, corresponding to the desired cooperativity-preserving parameterization.

Finally, the extension of all constraints towards the $H_{\infty}$ control design for the full augmented system model (38) follows by substituting the matrix $\mathbb{A}_{\mathrm{Q}}$ for the matrix $\mathcal{A}_{\mathrm{Q}}$ along with a corresponding redefinition of the output vector $\mathbf{y}_{\infty}(t)$ reflecting the requirements $\mathbf{C 1 a}, \mathbf{C} 1 \mathbf{b}, \mathbf{C} 1 \mathbf{c}$ (together replacing the criterion $\mathbf{C} 1$ ) as well as C2 and C3. For that purpose, the dimensions of all input and output matrices need to be adjusted appropriately. For additional details, especially with respect to an efficient numerical implementation, the reader is referred to [46].

Finally, it can be noticed that the worst-case simulation results in Fig. 5 for the overall cooperative system model and the experimentally measured unfiltered temperatures in Fig. 6 obtained at the available test rig — both implemented with a sampling interval of $0.1 \mathrm{~s}$ - are in very good coincidence. Hence, the observer-based feedback controller was validated successfully in terms of its capability to predict the domains of reachable states by a combination of a bank of interval observers with a cooperativity-preserving feedback control design. For all parameterization details, the reader is referred to [46], where the toolbox SEDUMI [51] in combination with YALMIP [29] was employed to solve all LMI-constrained optimization tasks.

Note, the computing times as well as the numbers of decision variables for the offline control parameterization are similar to the ones that are summarized for the observer design in Tab. 1. 


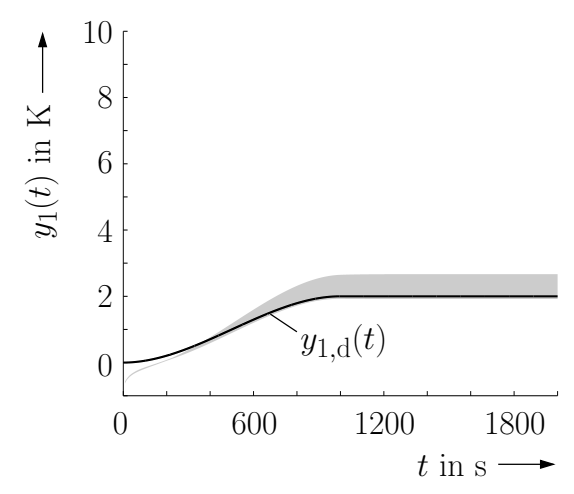

(a) System output $y_{1}(t)$.

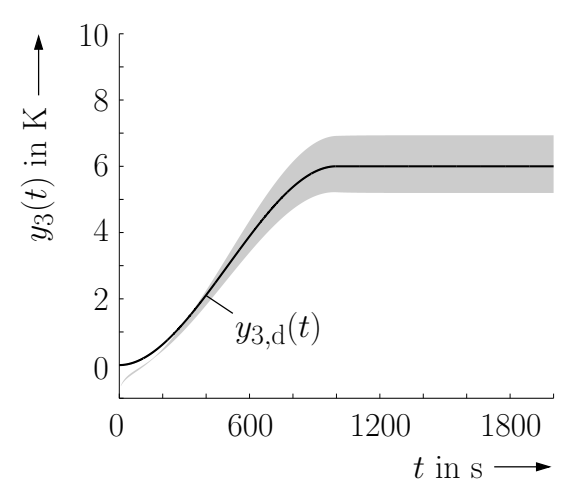

(c) System output $y_{3}(t)$.

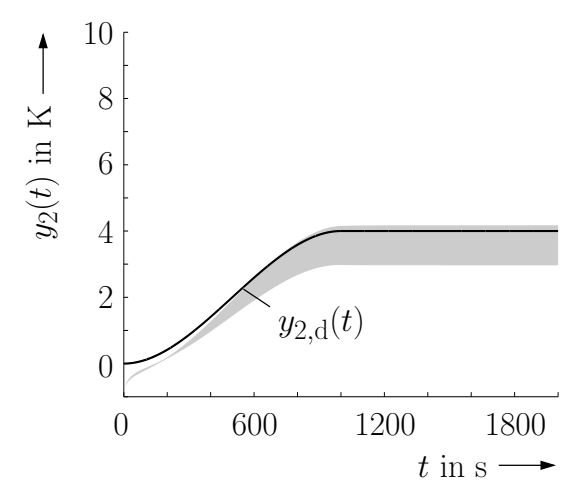

(b) System output $y_{2}(t)$.

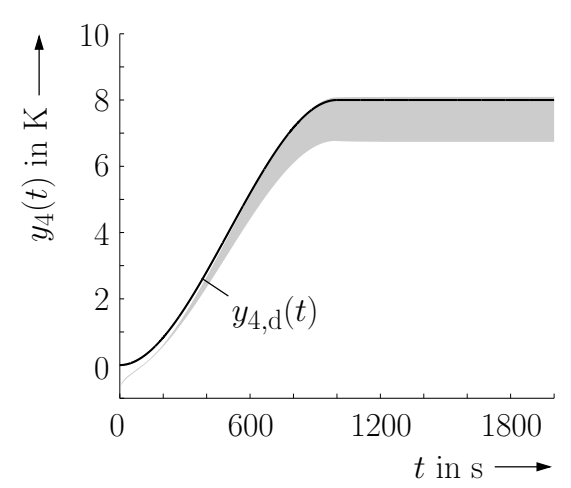

(d) System output $y_{4}(t)$.

Figure 5: Range bounds of the observer-based cooperative closed-loop controller for the uncertain system model in comparison with smooth reference trajectories for each of the $N=4$ rod segment midpoint temperatures under consideration of the same measurement tolerances of $\pm 0.75 \mathrm{~K}$ as in Sec. 2.5.

\section{Conclusions and Outlook on Future Work}

In this paper, the complete design procedure of cooperativity-preserving interval observer-based feedback controllers was described by means of a practical application scenario. For this scenario, advantages of optimized observer and control parameterizations which can be achieved by LMI-based formulations were compared with naive approaches which explicitly rely on the structural sign pattern of system, input, and output matrices for a specific control task at hand. In such a way, a design methodology was presented which removes the requirement for tedious trial-and-error parameterizations and leads to optimal observer and control structures which preserve the property of cooperativity if cooperativity already characterizes the open-loop dynamics at hand. 


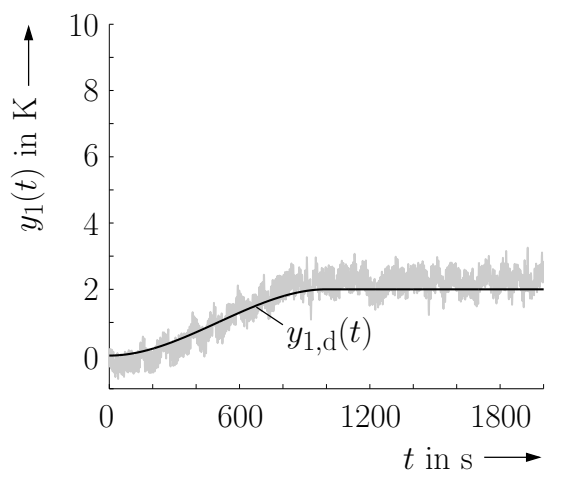

(a) System output $y_{1}(t)$.

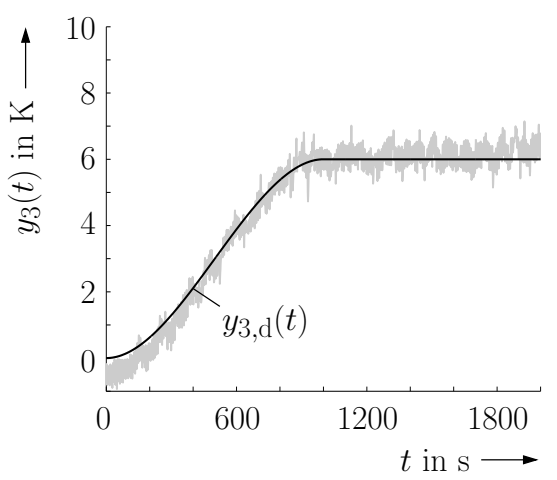

(c) System output $y_{3}(t)$.

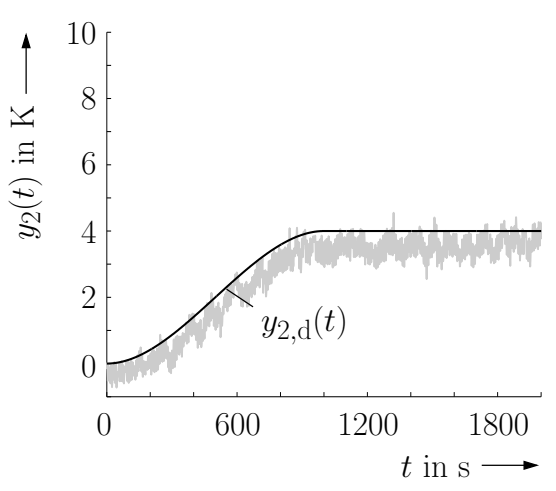

(b) System output $y_{2}(t)$.

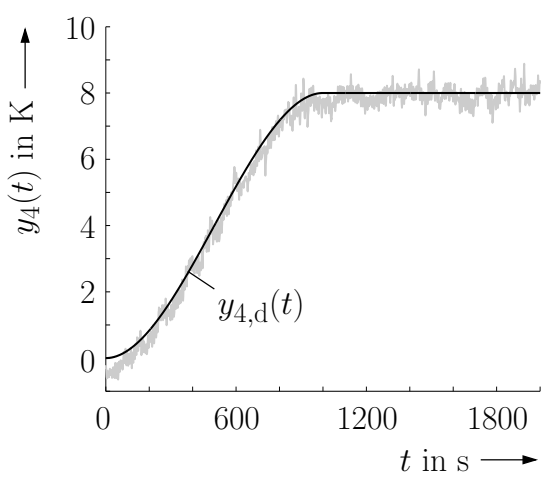

(d) System output $y_{4}(t)$.

Figure 6: Experimental results for the temperature tracking control at the midpoints of the $N=4$ rod segments.

Future work will deal with the design of partial state feedback approaches and output feedback controllers. These may lead to a significant reduction of the design as well as implementation effort if suitable input-output configurations can be determined with which both, robust stability despite parameter uncertainty and cooperativity of the closed-loop dynamics can be guaranteed.

\section{References}

[1] Alcaraz-Gonzlez, V., Steyer, J. P., Harmand, J., Rapaport, A., GonzlezAlvarez, V., and Pelayo-Ortiz, C. Application of a Robust Interval Observer to an Anaerobic Digestion Process. Developments in Chemical Engineering and Mineral Processing, 13(3-4):267-278, 2005. DOI: 10.1002/apj.5500130308. 
[2] Boyd, S., El Ghaoui, L., Feron, E., and Balakrishnan, V. Linear Matrix Inequalities in System and Control Theory. SIAM, Philadelphia, 1994. DOI: 10.1137/1.9781611970777.

[3] Chambon, E., Apkarian, P., and Burlion, L. Metzler Matrix Transform Determination using a Nonsmooth Optimization Technique with an Application to Interval Observers. In 2015 Proc. of the Conference on Control and its Applications, Paris, France, 2015. DOI: 10.1137/1.9781611974072.29.

[4] Csendes, T. and Ratz, D. Subdivision Direction Selection in Interval Methods for Global Optimization. SIAM Journal on Numerical Analysis, 34(3):922-938, 1997. DOI: 10.1137/S0036142995281528.

[5] Efimov, D., Li, S., Hu, Y., Muldoon, S., Javaherian, H., and Nikiforov, V. O. Application of Interval Observers to Estimation and Control of Air-Fuel Ratio in a Direct Injection Engine. In Proc. of American Control Conference (ACC), Chicago, IL, USA, 2015. DOI: 10.1109/ACC.2015.7170706.

[6] Efimov, D., Raïssi, T., Chebotarev, S., and Zolghadri, A. Interval State Observer for Nonlinear Time Varying Systems. Automatica, 49(1):200-205, 2013. DOI: $10.1016 / j$.automatica.2012.07.004.

[7] Gennat, M. and Tibken, B. Guaranteed Bounds for Uncertain Systems: Methods Using Linear Lyapunov-like Functions, Differential Inequalities and a Midpoint Method. In CD-Proc. of 12th GAMM-IMACS Intl. Symposium on Scientific Computing, Computer Arithmetic, and Validated Numerics SCAN 2006, Duisburg, Germany, 2007. IEEE Computer Society. DOI: 10.1109/SCAN. 2006.21.

[8] Guerra, P., Puig, V., and Witczak, M. Robust Fault Detection with UnknownInput Interval Observers using Zonotopes. In Proc. of IFAC World Congress, Seoul, Korea, 2008. DOI: 10.3182/20080706-5-KR-1001.00937.

[9] Hadj-Sadok, M.Z. and Gouz, J.-L. Bounds Estimations for Uncertain Models of Wastewater Treatment. In Proc. of the 1998 IEEE International Conference on Control Applications, Trieste, Italy, 1998. DOI: 10.1109/CCA.1998.728440.

[10] Hadj-Sadok, M.Z. and Gouz, J.-L. Comparison Between Two Interval Observers for Wastewater Treatment Models. In Proc. of European Control Conference, Karlsruhe, Germany, 1999.

[11] Ichalal, D., Marx, B., Maquin, D., and Ragot, J. State Estimation of System with Bounded Uncertain Parameters: Interval Multimodel Approach. International Journal of Adaptive Control and Signal Processing, 32:480-493, 2018. DOI: $10.1002 /$ acs. 2855.

[12] Ifqir, S., Ait-Oufroukh, N., Ichalal, D., and Mammar, S. Interval Observer for LPV Systems: Application to Vehicle Lateral Dynamics. In Proc. of IFAC World Congress, Toulouse, France, 2017. DOI: $10.1016 / j$. ifacol.2017.08.995. 
[13] Ifqir, S., Ait-Oufroukh, N., Ichalal, D., and Mammar, S. Synchronous Interval Observer Design for Switched LPV Systems Using Multiple Quadratic ISS-Lyapunov Functions. In Proc. of 25th Mediterranean Conference on Control and Automation (MED), Valletta, Malta, 2017. DOI: 10.1109/MED. 2017.7984149.

[14] Ifqir, S., Rauh, A., Kersten, J., Ichalal, D., Ait-Oufroukh, N., and Mammar, S. Interval Observer-Based Controller Design for Systems with State Constraints: Application to Solid Oxide Fuel Cells Stacks. In IEEE Intl. Conference on Methods and Models in Automation and Robotics MMAR 2019, Miedzyzdroje, Poland, 2019. DOI: 10.1109/MMAR.2019.8864718.

[15] Jaulin, L., Kieffer, M., Didrit, O., and Walter, É. Applied Interval Analysis. Springer-Verlag, London, 2001. DOI: 10.1007/978-1-4471-0249-6.

[16] Kaczorek, T. Positive 1D and 2D Systems. Springer-Verlag, London, 2002. DOI: $10.1007 / 978-1-4471-0221-2$.

[17] Kersten, J., Rauh, A., and Aschemann, H. State-Space Transformations of Uncertain Systems with Purely Real and Conjugate-Complex Eigenvalues into a Cooperative Form. In IEEE Intl. Conference on Methods and Models in Automation and Robotics MMAR 2018, Miedzyzdroje, Poland, 2018. DOI: 10.1109/MMAR . 2018.8486085.

[18] Kharkovskaia, T., Efimov, D., Fridman, E., Polyakov, A., and Richard, J.-P. On Design of Interval Observers for Parabolic PDEs. In Proc. of 20th IFAC World Congress, Toulouse, France, 2017. DOI: 10.1016/j. ifacol.2017.08.723.

[19] Kharkovskaya, T., Efimov, D., Polyakov, A., and Richard, J.-P. Interval Observers for PDEs: Approximation Approach. In Proc. of 10th IFAC Symposium on Nonlinear Control Systems (NOLCOS), Monterey, USA, 2016. DOI: 10.1016/j.ifacol.2016.10.283.

[20] Kharkovskaya, T., Efimov, D., Polyakov, A., and Richard, J.-P. Design of Interval Observers and Controls for PDEs Using FiniteEelement Approximations. Automatica, 93:302-310, 2018. DOI: $10.1016 / j$. automatica. 2018.03.016.

[21] Kletting, M., Rauh, A., Aschemann, H., and Hofer, E.P. Interval Observer Design for Nonlinear Systems with Uncertain Time-Varying Parameters. In Proc. of 12th IEEE International Conference on Methods and Models in Automation and Robotics MMAR, pages 361-366, Miedzyzdroje, Poland, 2006.

[22] Krasnochtanova, I., Rauh, A., Kletting, M., Aschemann, H., Hofer, E.P., and Schoop, K.-M. Interval Methods as a Simulation Tool for the Dynamics of Biological Wastewater Treatment Processes with Parameter Uncertainties. Applied Mathematical Modeling, 34(3):744-762, 2010. DOI: $10.1016 / j . a p m .2009 .06 .019$. 
[23] Krawczyk, R. Newton-Algorithmen zur Bestimmung von Nullstellen mit Fehlerschranken. Computing, 4:189-201, 1969. DOI: 10.1007/BF02234767, In German.

[24] Krebs, S., Bchle, M., and Hohmann, S. Coupled Boundary Interval Observer for LPV Systems Subject to Uncertainties in Input, Output and Parameters. Automatica, 95:426-432, 2018. DOI: 10.1016/j.automatica.2018.06.012.

[25] Krebs, S., Gellrich, T., and Hohmann, S. Interval Observers for LPV Systems and Application to the Guaranteed State Estimation of an Induction Machine. In Proc. of IFAC World Congress, Toulouse, France, 2017. DOI: 10.1016/j.ifacol.2017.08.629.

[26] Lendek, Z., Guerra, T.-M., Babuska, R., and De Schutter, B. Stability Analysis and Nonlinear Observer Design Using Takagi-Sugeno Fuzzy Models, volume 262. 01 2011. DOI: 10.1007/978-3-642-16776-8.

[27] Li, J., Wang, Z., Shen, Y., and Wang, Y. Interval Observer Design for DiscreteTime Uncertain TakagiSugeno Fuzzy Systems. IEEE Transactions on Fuzzy Systems, 27(4):816-823, 2019. DOI: 10.1109/TFUZZ.2019.2898161.

[28] Lin, Y. and Stadtherr, M.A. Validated Solutions of Initial Value Problems for Parametric ODEs. volume 57, pages 1145-1162, 2007. DOI: 10.1016/j. apnum.2006.10.006.

[29] Löfberg, J. YALMIP: A Toolbox for Modeling and Optmization in MATLAB. In Proc. of IEEE Intl. Symposium on Computer Aided Control Systems Design, pages 284-289, Taipei, Taiwan, 2004. DOI: 10.1109/CACSD . 2004.1393890.

[30] Lohner, R. Enclosing the Solutions of Ordinary Initial and Boundary Value Problems. In Kaucher, E. W., Kulisch, U. W., and Ullrich, C., editors, Computer Arithmetic: Scientific Computation and Programming Languages, pages 255-286, Stuttgart, 1987. Wiley-Teubner Series in Computer Science.

[31] Lohner, R. On the Ubiquity of the Wrapping Effect in the Computation of the Error Bounds. In Kulisch, U., Lohner, R., and Facius, A., editors, Perspectives on Enclosure Methods, pages 201-217, Wien, New York, 2001. Springer-Verlag. DOI: $10.1007 / 978-3-7091-6282-8 \_12$.

[32] Martínez García, C., Puig, V., and Astorga-Zaragoza, C.-M. Fault Estimation Using a Takagi-Sugeno Interval Observer: Application to a PEM Fuel Cell. In Proc. of ICINCO, Madrid, Spain, 2017. DOI: 10.5220/0006431206130620.

[33] Martnez Garca, C., Puig, V., Astorga-Zaragoza, C.M., and Osorio-Gordillo, G.L. Robust Fault Estimation Based on Interval Takagi-Sugeno Unknown Input Observer. In Proc. of 10th IFAC Symposium on Fault Detection, Supervision and Safety for Technical Processes SAFEPROCESS 2018, Warsaw, Poland, 2018. DOI: 10.1016/j.ifacol.2018.09.624. 
[34] Mazenc, F., Andrieu, V., and Malisoff, M. Design of Continuous-Discrete Observers for Time-Varying Nonlinear Systems. Automatica, 57(Supplement C):135-144, 2015. DOI: 10.1016/j.automatica. 2015.04.016.

[35] Mazenc, F. and Bernard, O. Asymptotically Stable Interval Observers for Planar Systems With Complex Poles. IEEE Transactions on Automatic Control, 55(2):523-527, Feb 2010. DOI: 10.1109/TAC. 2009.2037472.

[36] Menasria, Y., Bouras, H., and Debbache, N. An Interval Observer Design for Uncertain Nonlinear Systems Based on the T-S Fuzzy Model. Archives of Control Sciences, 27:307-407, 2017. DOI: 10.1515/acsc-2017-0025.

[37] Nedialkov, N. S. Interval Tools for ODEs and DAEs. In CD-Proc. of 12th GAMM-IMACS Intl. Symposium on Scientific Computing, Computer Arithmetic, and Validated Numerics SCAN 2006, Duisburg, Germany, 2007. IEEE Computer Society. DOI: 10.1109/SCAN.2006.28.

[38] Neumaier, A. Interval Methods for Systems of Equations. Cambridge University Press, Encyclopedia of Mathematics, Cambridge, 1990. DOI: 10.1017/CB09780511526473.

[39] Puig, V., Saludes, J., and Quevedo, J. Worst-Case Simulation of Discrete Linear Time-Invariant Interval Dynamic Systems. Reliable Computing, 9(4):251290, 2003. DOI: $10.1023 / \mathrm{A}: 1024666428387$.

[40] Raïssi, T. and Efimov, D. Some Recent Results on the Design and Implementation of Interval Observers for Uncertain Systems. at-Automatisierungstechnik, 66(3):213-224, 2018. DOI: 10.1515/auto-2017-0081.

[41] Raïssi, T., Efimov, D., and Zolghadri, A. Interval State Estimation for a Class of Nonlinear Systems. IEEE Transactions on Automatic Control, 57:260-265, 2012. DOI: $10.1109 / \mathrm{TAC} .2011 .2164820$.

[42] Rauh, A., Kersten, J., and Aschemann, H. An Interval Approach for Parameter Identification and Observer Design of Spatially Distributed Heating Systems. In Proc. of 9th Vienna International Conference on Mathematical Modelling MATHMOD 2018, Vienna, Austria, 2018. DOI: $10.1016 / j$. ifacol.2018.03.058.

[43] Rauh, A., Kersten, J., and Aschemann, H. An Interval Observer Approach for the Online Temperature Estimation in Solid Oxide Fuel Cell Stacks. In Proc. of European Control Conference ECC, Limassol, Cyprus, 2018. DOI: 10.23919/ECC. 2018.8550158.

[44] Rauh, A., Kersten, J., and Aschemann, H. Intervallmethoden fr Identifikation, Beobachter- und Reglersynthese von Finite-Volumen-Modellen thermischer Prozesse (Interval Methods for the Identification as well as Observer and Control Design for Finite-Volume Models of Thermal 
Processes). at-Automatisierungstechnik, 66(8):633-646, 2018. DOI: 10.1515/auto-2017-0117, In German.

[45] Rauh, A., Kersten, J., and Aschemann, H. Linear Matrix Inequality Techniques for the Optimization of Interval Observers for Spatially Distributed Heating Systems. In IEEE Intl. Conference on Methods and Models in Automation and Robotics MMAR 2018, Miedzyzdroje, Poland, 2018. DOI: 10.1109/MMAR. 2018.8486120.

[46] Rauh, A., Kersten, J., and Aschemann, H. Interval and Linear Matrix Inequality Techniques for Reliable Control of Linear Continuous-Time Cooperative Systems with Applications to Heat Transfer. International Journal of Control, pages 1-18, 2020. DOI: 10.1080/00207179.2019.1708966, Available online.

[47] Rauh, A., Kersten, J., and Aschemann, H. Interval Methods and ContractorBased Branch-and-Bound Procedures for Verified Parameter Identification of Quasi-Linear Cooperative System Models. Journal of Computational and Applied Mathematics, 367:112484, 2020. DOI: 10.1016/j.cam.2019.112484.

[48] Sauvage, F., Dochain, D., and Monge, T. Design of an Interval Observer for Exothermic Fed-Batch Processes. In Proc. of 8th IFAC Symposium on Dynamics and Control of Process Systems, Cancn, Mexico, 2007. DOI: 10.3182/20070606-3-MX-2915.00059.

[49] Scherer, C. and Weiland, S. Linear Matrix Inequalities in Control. In Levine, W. S., editor, Control System Advanced Methods, The Electrical Engineering Handbook Series, pages 24-1-24-30. CRC Press, Boca Raton, 2nd edition, 2011. DOI: $10.1201 / \mathrm{b} 10384$.

[50] Smith, H. L. Monotone Dynamical Systems: An Introduction to the Theory of Competitive and Cooperative Systems, volume 41. Mathematical Surveys and Monographs, American Mathematical Soc., Providence, 1995.

[51] Sturm, J. F. Using SeDuMi 1.02, A MATLAB Toolbox for Optimization over Symmetric Cones. Optimization Methods and Software, 11-12(1-4):625-653, 1999. DOI: $10.1080 / 10556789908805766$.

[52] Takagi, T. and Sugeno, M. Fuzzy Identification of Systems and Its Applications to Modeling and Control. IEEE Transactions on Systems, Man, and Cybernetics, SMC-15(1):116-132, 1985. DOI: 10.1109/TSMC.1985.6313399.

[53] Weinmann, A. Uncertain Models and Robust Control. Springer-Verlag, Wien, 1991. DOI: $10.1007 / 978-3-7091-6711-3$. 